\title{
Enhancing the electrocatalytic activity and stability of Prussian Blue Analogues through the introduction of Au nanoparticles in a core@shell heterostructure
}

\author{
Roger Sanchis-Gual, Toribio F. Otero, Marc Coronado-Puchau, * Eugenio Coronado* \\ R. Sanchis-Gual, Dr. M. Coronado-Puchau, Prof. E. Coronado \\ Instituto de Ciencia Molecular (ICMol) \\ Universidad de Valencia, \\ C/ Catedrático José Beltrán 2, Paterna, 46980, Spain \\ E-mail:marc.coronado@uv.es, eugenio.coronado@uv.es \\ Prof. T. F. Otero \\ Centre for Electrochemistry and Intelligent Materials (CEMI) \\ Universidad Politécnica de Cartagena \\ Aulario II, Paseo Alfonso XIII, E-30203 Cartagena, Murcia, Spain
}

Keywords: core@shell nanoparticle, Prussian Blue Analogue, Au nanoparticle, electrocatalyst, oxygen evolution reaction

Prussian blue analogues (PBAs) have shown to be useful as earth-abundant electrocatalysts for the Oxygen Evolution Reaction (OER) in acidic, neutral and alkaline media. Still, further improvements can be achieved by increasing their electrical conductivity. In this work, we have obtained and fully characterized a variety of monodisperse core@ shell hybrid nanoparticles of Au@PBA (PBA of $\mathrm{Ni}^{\mathrm{II}} \mathrm{Fe}^{\mathrm{II}}$ and $\mathrm{Co}^{\mathrm{II}} \mathrm{Fe}^{\mathrm{II}}$ ) with different shell sizes. Their electrocatalytical activity is evaluated by studying the OER, which is compared to the pristine PBA and other Au-PBA heterostructures. It was observed that the introduction in a core@shell of 5-10\% of Au in weight leads to an increment in the electroactive mass able to be reduced or oxidized and thus, to a higher number of sites capable to take part in the OER. This larger amount of electroactive sites leads to a significant decrease in the onset potential (a reduction of the onset potential up to $100 \mathrm{mV}$ and an increase up to $420 \%$ of the current density recorded at an overpotential of $350 \mathrm{mV}$ ), while the Tafel slope remains unchanged, suggesting that Au reduces the limiting potential of the catalyst with no variation in the reaction kinetics. These effects are not experimented in the other Au-PBA nanostructures mainly due to the lower contact between both compounds and the oxidation of Au. Hence, an Au core activates the PBA shell and 
increases the conductivity of the resulting hybrid while the PBA shell prevents Au oxidation. These improvements come from the strong synergistic effect existing in the core@shell structure and evidence the importance of the chemical design for preparing PBA-based nanostructures displaying better electrocatalytic performances and higher electrochemical stabilities.

\section{Introduction}

The electrolysis of water to produce hydrogen and oxygen offers the exciting possibility of storing energy whose importance is out of question today. However, this process requires high voltages mainly due to the slow kinetics of the oxygen evolution reaction (OER). This reaction represents a significant efficiency loss in water-splitting systems and often requires anode catalyst to reduce the energy barriers of the OER. In this context, earth-abundant electrocatalysts have been extensively studied in alkaline media. ${ }^{[1-3]}$ First-row transition metal oxides of $\mathrm{Co}, \mathrm{Fe}$, or Ni show extremely high activities but only at very high pH (above 13), where the corresponding half-cell reduction reaction (for example, the hydrogen production) is more difficult. Nevertheless, at lower $\mathrm{pH}$, most of the catalysts suffer corrosion. Thus, an efficient catalyst for OER at acidic or near-neutral solution based on earth-abundant metals remains an important challenge. In such conditions, benchmark materials are principally based on Ir or Ru oxides, affecting considerably the cost of this technology. ${ }^{[4,5]}$

Prussian blue analogues (PBA) are coordination polymers based on bimetallic cyanide complexes of general formula $\mathrm{A}_{\mathrm{a}} \mathrm{B}_{\mathrm{b}}\left[\mathrm{D}(\mathrm{CN})_{6}\right]_{\mathrm{d}}(\mathrm{A}=$ alkali cation, $\mathrm{B}$, and $\mathrm{D}=$ transition metal ions). ${ }^{[6]}$ These materials are of interest in this context since they are robust, chemically tuneable, based on earth-abundant metals and they exhibit large specific surface areas. In fact, they have recently been investigated as an interesting implementation in multifunctional materials for many energy-related fields, such as supercapacitors, sodium-ion batteries and electrocatalysts. ${ }^{[7-10]}$ Moreover, well-stabilized PBAs are robust and stable in a large $\mathrm{pH}$ range 
exhibiting catalytic activities comparable to those of metal oxides for OER. ${ }^{[10]}$ However, PBAs exhibit low electrical conductivities, which increase the required overpotentials in water splitting. ${ }^{[11]}$ For this reason, the combination of PBA with other nanomaterials has attracted considerable attention in order to further improve their electrocatalytic activity. ${ }^{[10,12]}$

Recent studies have shown that electrocatalysts based on $\mathrm{Co}$, Ni and $\mathrm{Fe}$ are oxidized before the onset of oxygen evolution, being the oxidized metals the active species in the pathway for $\mathrm{O}_{2}$ evolution. ${ }^{[13-15]}$ Thus, a possibility to enhance the electrocatalytic activity could be done by combining efficient electrocatalysts with highly electronegative metals. In this line, Au is the highest electronegative metal and thus it can drain electrons from the catalyst. ${ }^{[16,17]}$ Besides, in combination with the electrocatalyst it may contribute to improve the electrocatalytic performance thanks to the increase in the conductivity of the resulting material. ${ }^{[18-20]}$ To this aim, in this work we have explored the preparation of hybrid materials combining PBAs with Au. In particular, we have focused on nanoparticles (NPs) since they are usually the preferred form of catalysts due to the large number of electroactive sites provided by their high surface areas. Among them, the core@shell structure has been extensively used to obtain hybrid materials at the nanometer scale. ${ }^{[21,22]}$ Still, Au@PBA heterostructure remains an unexplored hybrid material for electrocatalysis. Thus, Au@PBA heterostructures have been mostly focused on the pure Prussian blue compound $\left(\mathrm{A}_{\mathrm{a}} \mathrm{Fe} \mathrm{x}_{\mathrm{x}}\left[\mathrm{Fe}(\mathrm{CN})_{6}\right]_{\mathrm{y}}\right) .{ }^{[23-27]}$ Only recently, heterostructures with other metallic compositions such as $\mathrm{NiFe}$ and $\mathrm{CoFe}$, which are interesting for OER, have been reported. ${ }^{[28,29]}$

The aim of this work is to study the influence of Au on the electrocatalytical activity of PBA. To do so, we have synthesized and fully characterized a variety of monodisperse core@ shell NPs of Au@PBA (PBA of $\mathrm{Ni}^{\mathrm{II}} \mathrm{Fe}^{\mathrm{II}}$ and $\mathrm{Co}^{\mathrm{II}} \mathrm{Fe}^{\mathrm{II}}$ ) with an $\mathrm{Au}$ core diameter of $c a .15 \mathrm{~nm}$ and different shell sizes. The OER of these NPs has been systematically evaluated and compared to that of the pristine PBA and other Au-PBA heterostructures. By means of different techniques, we have analyzed the electroactive mass able to be oxidized or reduced, the active surface sites 
and the resistances associated with the OER. We demonstrate that the existence of a strong synergistic effect between the core and the shell results in a large enhancement of the electrochemical performance and electrochemical stability of the hybrid Au@PBA heterostructures.

\section{Preparation and characterization of the Au@PBA nanoparticles}

\subsection{Synthesis}

The preparation of core@shell NPs of Au@PBA involves a two-step protocol in which $\mathrm{KAu}(\mathrm{CN})_{2}$ reacts with $\mathrm{KBH}_{4}$ in aqueous solution, reducing $\mathrm{Au}(\mathrm{I})$ to metallic $\mathrm{Au}$, to afford $\mathrm{Au}$ NPs stabilized by cyanide groups (Au@CN NPs), followed in a second step by a dropwise addition of the PBA precursors to overgrow the PBA around the Au surface. ${ }^{[28,29]}$ Still, this protocol needs further optimization to fulfill the requirements that are needed to make these hybrid Au@PBA NPs useful for electrocatalysis: (i) the amount of Au should be minimized with respect to that of the electroactive PBA shell since one needs to have the maximum number of electroactive sites while maintaining the beneficial properties of the $\mathrm{Au}$ core in terms of conductivity and activation of the shell (see below); (ii) a good coverage of the Au by the PBA shell to avoid its oxidation and dissolution during the electrochemical process; (iii) a good monodispersity in the size of the hybrid NPs to ensure the reproducibility in the electrochemical measurements.

With these considerations in mind, the first step in the preparation of Au@PBA NPs has been carried out at low temperature (around $10^{\circ} \mathrm{C}$ ). This temperature was set to induce the formation of smaller Au NPs and, consequently, to increase the surface area and the contact between $\mathrm{Au}$ and PBA. These NPs are in an equilibrium between borohydride (reducing agent) and cyanide (capping but also oxidizing agent). Furthermore, since $\mathrm{KBH}_{4}$ leads to hydrogen bubbles that destabilize the Au NPs as a consequence of the water reduction, ${ }^{[30]}$ in our protocol the $\mathrm{Au}(\mathrm{CN})_{2}: \mathrm{KBH}_{4}$ molar ratio was increased with respect to the original synthesis (from 0.08 to 0.25) to improve the Au monodispersity. This smaller NP size distribution was proved by the 
width of the Au plasmon band (see below). On the other hand, in the specific case of $\mathrm{Au} @ \mathrm{CoFe}$ NPs, some additional changes need to be introduced to improve the CoFe coverage. Indeed, in this growth process, $\mathrm{CoFe}$ has a stronger tendency to self-nucleate than $\mathrm{NiFe}$, as a consequence of the different kinetics. ${ }^{[31]}$ For this reason, following the original protocol afforded an incomplete coverage of the Au NP by the overgrown shell (Figure S1A). To solve this problem, the precursors were added at lower rates in order to give enough time to the CoFe shell to grow better over Au (Figure S1B). Although a core@shell morphology is achieved at this point, a further improvement was still by varying the time delay (time between Au NPs formation and the beginning of the addition of the precursors). Thus, a higher time delay promotes the formation of single CoFe NPs (Figure S1C), while no time delay prevents the formation of well-stabilized Au@CN NPs (Figure S1D). A time delay of 10-20 min in combination with an addition rate of $0.5 \mathrm{~mL} \cdot \mathrm{h}^{-1}$ appears to be the most optimal conditions to get well stabilized $\mathrm{Au}$ NPs and thus, a well-defined shell growth around the metallic surface. Furthermore, PBA shell thickness can be controlled by varying the total PBA precursor volumes. Thus, after optimizing the protocol, we synthesized NPs perfectly covered by two limit shell sizes (i.e. a thin and a thick shell) to study their electrochemical performance (Figure 1). 

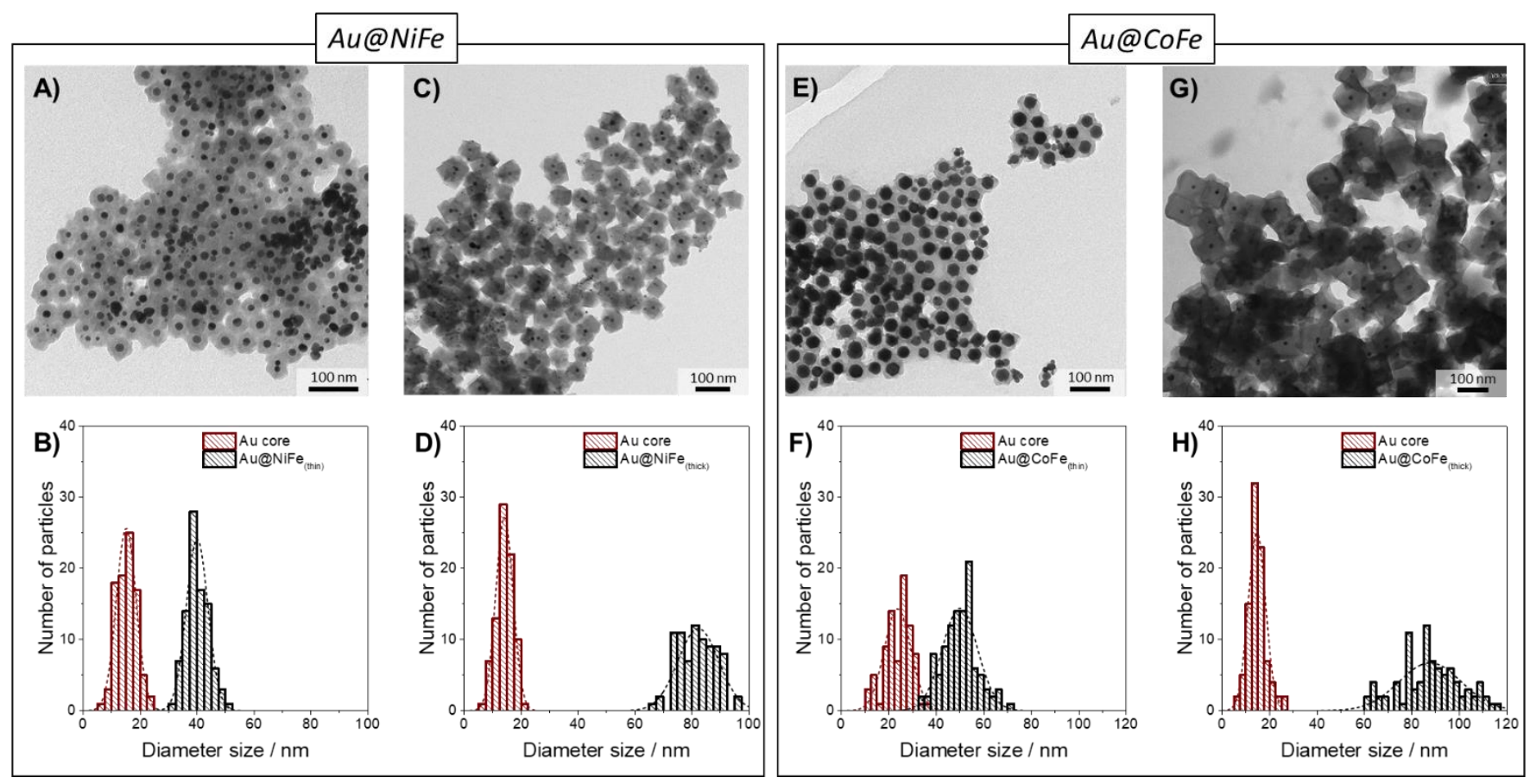

Figure 1. TEM images and histograms of the size distribution for Au@NiFe NPs with thinner (A, B) and thicker shells (C, D) and for Au@CoFe with thinner (E, F) and thicker shells $(\mathrm{G}, \mathrm{H})$.

\subsection{Physical characterization}

TEM images of the hybrid NPs show a core@shell morphology formed by an Au core and a well-defined PBA shell (Figure 1). For NiFe, hybrid NPs with shell thicknesses of $12 \pm 2$ and $30 \pm 3 \mathrm{~nm}$ were prepared, while maintaining a constant size of about $15 \mathrm{~nm}$ for the Au core (Figure 1A to 1D). For CoFe, shell thicknesses of $13 \pm 3$ and $35 \pm 6 \mathrm{~nm}$ have been obtained, but, in contrast to those of $\mathrm{NiFe}$, the size of the Au core varies from 22 to $15 \mathrm{~nm}$, respectively (Figure 1E to $1 \mathrm{H})$. This core size difference could be a consequence of the major difficulties encountered in the CoFe system for growing the PBA layer around Au.

Interestingly, the Localized Surface Plasmon Resonance (LSPR) of the Au NPs is progressively red-shifted during shell formation (Figure S2). It can be attributed to the progressive change in the dielectric constant around the Au surface upon the continuous overgrowth of the shell. ${ }^{[32,33]}$ It is expected that $\mathrm{NiFe}$ and $\mathrm{CoFe}$ exhibit quite similar values for the dielectric constant. Indeed, by comparing the red-shift shown by Au@PBA NPs with different shell thicknesses, one can 
notice that for those having a $12-13 \mathrm{~nm}$ shell, a shift of $c a .21 \mathrm{~nm}$ is observed, while for those having a 30-35 $\mathrm{nm}$ shell this shift turns out to be of $c a .28 \mathrm{~nm}$. In addition, the full width at half maximum (FWHM) of the plasmon band is slightly reduced after the PBA overgrowth. In point of fact, the modification in the dielectric constant also leads to an increase in the plasmon band intensity that decreases the FWHM (Figure S2). Moreover, the absence of other plasmon bands together with the small width of these bands point toward a narrow size distribution for the $\mathrm{Au}$ NPs. These plasmonic properties could be also beneficial for increasing the catalytic performance by light irradiation since the increase in the local temperature could reduce even more the onset potential and enhance the reaction rate. ${ }^{[34,35]}$

Energy-dispersive X-ray spectroscopy (EDX) demonstrates a homogeneous distribution of iron and nickel, as well as iron and cobalt, over the Au NPs (see Figure S3). The estimated gold content for the thinner and thicker shells is $c a .40 \%$ and $c a .8 \%$, respectively (see Table S1). The molecular formulas estimated by Inductively Coupled-Plasma Optical Emission Spectrometry (ICP-OES) can be found in the Supporting Information (Table S2). It is important to note that these PBAs do not contain many vacancies (thus, few structural defects are present ${ }^{[36]}$ ) since $\mathrm{KBH}_{4}$ guarantees a high amount of potassium cations in the solution.

X-ray photoelectron spectroscopy (XPS) was performed on a powder of these NPs to get information on the oxidation state of the metals contained in the PBA shells. For Au@NiFe, nickel is present as $\mathrm{Ni}^{\mathrm{II}}$, while iron is reduced from $\mathrm{Fe}^{\mathrm{III}}$ to $\mathrm{Fe}^{\mathrm{II}}$ (Figure $\mathrm{S} 4$ ). This is in good agreement with previous works reported in the literature ${ }^{[28]}$ and with the magnetic measurements. Indeed, while pristine $\mathrm{Ni}^{\mathrm{II}} \mathrm{Fe}{ }^{\mathrm{III}} \mathrm{PBA}$ behaves as a magnet below $c a .20 \mathrm{~K},{ }^{[37]}$ the $\mathrm{Au} @ \mathrm{NiFe}$ NPs stay paramagnetic down to $2 \mathrm{~K}$ (Figure S5) as a consequence of the reduction of $\mathrm{Fe}^{\mathrm{III}}$ (with $\mathrm{S}=1 / 2$ ) to $\mathrm{Fe}^{\mathrm{II}}$ (with $\mathrm{S}=0$ ). Similarly, while pristine $\mathrm{Co}^{\mathrm{II}} \mathrm{Fe}^{\mathrm{III}}$ PBA exhibits magnetic ordering below $14 \mathrm{~K},{ }^{[37]} \mathrm{Au} @ \mathrm{CoFe}$ remains paramagnetic at lower temperatures (Figure S5). Such a reduction is promoted by $\mathrm{KBH}_{4}$, which is still present in the solution. A high concentration of this strong reductant is required to reach an equilibrium between the $\mathrm{Au}$ 
reduction and the oxidation by cyanide. Lower amounts lead to non-stabilized Au NPs that can precipitate. Even though the presence of $\mathrm{Fe}^{\mathrm{II}}$, the $\mathrm{Au} @ \mathrm{PBA}$ heterostructures maintain the characteristic PB cubic structure in the XRDP data and the expected cyanide vibration in the Raman spectra, located at ca. $2150 \mathrm{~cm}^{-1}$ (see Figure 2).
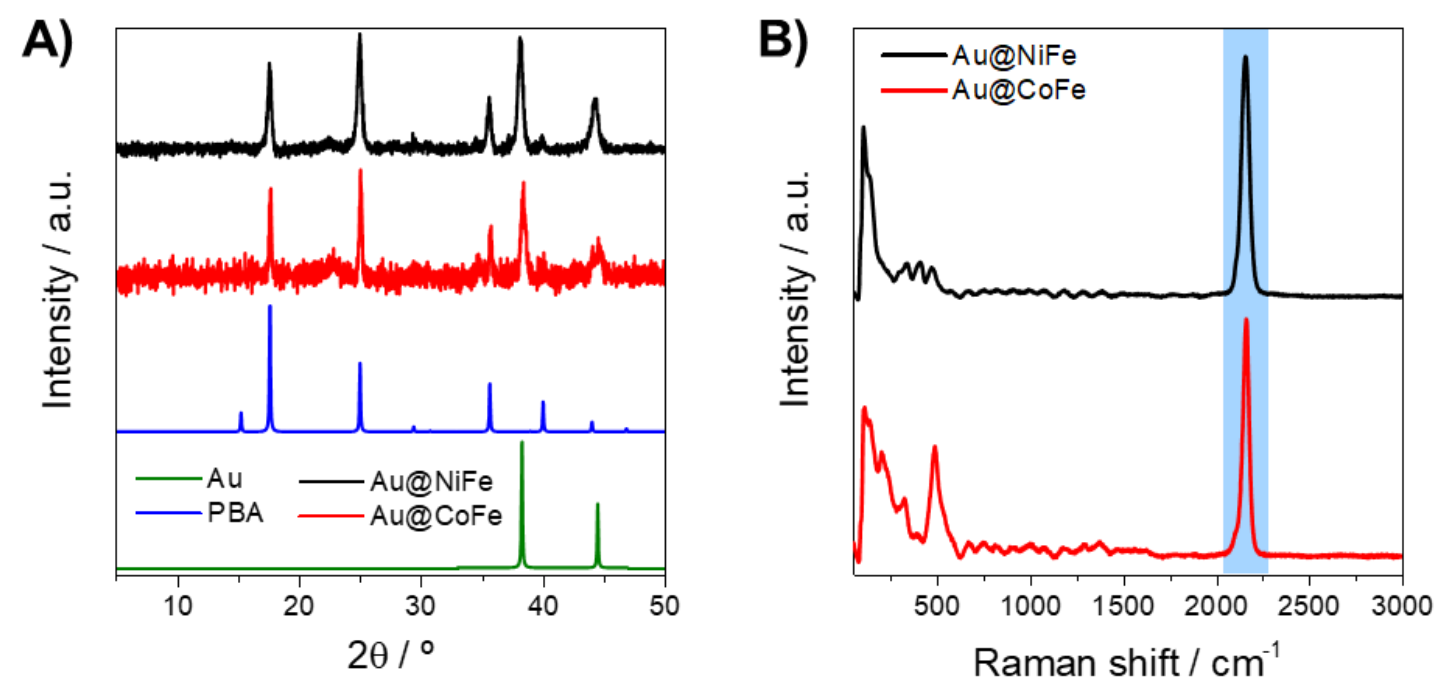

Figure 2. A) XRDP and B) Raman spectrum of the Au@ PBA NPs. The cyanide vibration peak is highlighted in blue.

\section{Electrochemical characterization}

To verify the influence of $\mathrm{Au}$ on the electrochemical performance and the benefits of this sort of core@shell heterostructure, four kinds of NPs were also prepared: i) PBA NPs (NiFe ${ }^{\mathrm{III}}$ and $\mathrm{CoFe}^{\mathrm{III}}$ ) containing many defects (i.e. many vacancies) since they give rise to higher electrocatalytic performance, ${ }^{[38]}$ ii) PBA with reduced $\mathrm{Fe}^{\mathrm{II}}\left(\mathrm{NiFe}^{\mathrm{II}}\right.$ and $\left.\mathrm{CoFe}^{\mathrm{II}}\right)$ iii) PBA NPs decorated with $\mathrm{Au}$ NPs on its surface $(\mathrm{Au}-\mathrm{PBA})^{[39]}$ and PBA NPs physically mixed with $\mathrm{Au}$ NPs (Au+PBA). TEM images of these systems and their respective histograms can be found in Figures S6-S9.

\subsection{Evaluation of the Coulovoltammetric response}


The different abovementioned nanostructures were tested as OER electrocatalysts. Prior to that, CV curves were examined to analyze the redox processes. The Au@NiFe NPs exhibit redox peaks in a similar position as those observed for PBA-NiFe NPs of similar size (around 1.2 $1.5 \mathrm{~V}$ ). These peaks are related to Ni processes (Figure S10). ${ }^{[40]}$ In the CoFe case, all the studied NPs show a small redox couple between 1.2 and $1.4 \mathrm{~V}$, which can be assigned to Co redox processes (Figure S11) ${ }^{[41]}$ Observing the dependence of the charge on the applied potential, it can be noticed that NiFe compounds display almost reversible peaks (closed coulovoltammetric loops $;{ }^{[2,43]}$ Figure S12), whereas, in CoFe compounds, an excess anodic charge was observed (open coulovoltammetric loops $;{ }^{[42,43]}$ Figure S13). This irreversible charge comes from the lower onset potential of the OER; this leads to the irreversible formation of $\mathrm{O}_{2}$ at the most anodic potential. As it is demonstrated here, this is a useful technique since it brings the possibility to separate charges consumed by electrodic redox processes from charges expended by irreversible electrocatalytic processes — as oxygen evolution here - when both processes overlap in the same potential range. Therefore, this technique can provide a deeper understanding of both the onset OER potential and the evolution of the oxygen production. ${ }^{[44]}$ In this work, coulovoltammetry has been used to extract and analyze the reversible charges of the different compounds.

Remarkably, important differences in the current density and reversible charge are observed in the NPs, especially for the NiFe systems (Figures S10-S13). Thus, compared to the pristine $\mathrm{NiFe}^{\mathrm{III}}$ and $\mathrm{NiFe}^{\mathrm{II}}$, the system $\mathrm{Au} @ \mathrm{NiFe}$ with a thin shell displays lower current density values, possibly due to the low content of electroactive NiFe material, which represents $c a .60 \%$ of the total mass. In fact, if one takes into account the electroactive mass for these three cases, the current density and the total charge coming from these redox processes are quite similar (see Figure S14), suggesting that the density values are controlled by the percentage of electroactive PBA. Interestingly, this rule is not followed for Au@NiFe NPs with a thick shell. Then, with around $90 \%$ (in weight) of $\mathrm{NiFe}$, the current densities are about 5 times greater for the 
core@shell NPs as compared with those of the NiFe ${ }^{\mathrm{II}}$ NPs and even with those of the pristine $\mathrm{NiFe}^{\mathrm{III}} \mathrm{NPs}$. This strong and anomalous enhancement in the current density could be related to the increase in the conductivity of the hybrid material (compared with that of pristine PBA NPs), as well as with the number of electroactive sites. In this line, using Faraday's law of electrochemical reactions and the redox charges extracted from Figures S12 and S13, the total PBA mass involved in the redox processes was estimated. For pristine PBA, the PBA of Fe ${ }^{\mathrm{II}}$ and Au@PBA(thin), it was found that the reduced or oxidized PBA mass is below $10 \%$ of the total PBA electrodic mass (See Table S3), suggesting that only the surface of the PBA material is electrochemically active. Higher electroactive percentages of the PBA mass were estimated for the core@ shell heterostructures with a thick shell. Indeed, compared to the PBA of Fe ${ }^{\mathrm{II}}$, in the case of $\mathrm{Au} @ \mathrm{NiFe}($ thick), an increment in the mass of electroactive species of around $600 \%$ was calculated, while for the Au@CoFe(thick) system, this increment was found to be around $300 \%$. These results are in good agreement with the increase of the electrochemical surface area (ECSA) for these compounds (Figure S15). Notice that the ECSA represents the number of electroactive sites in the catalyst. ${ }^{[45]}$ Some studies have indicated that Au contributes remarkably to the enhancement of this parameter. ${ }^{[46]}$ In our case and compared to the PBA of $\mathrm{Fe}^{\mathrm{II}}$, an increment in the number of electroactive species of around $350 \%$ was calculated for $\mathrm{Au} @ \mathrm{NiFe}$ (thick), while this increment was found to be around $500 \%$ for the $\mathrm{Au} @ \mathrm{CoFe}$ (thick) system. Here the role of $\mathrm{Au}$ is the activation of the PBA shell, facilitating the interaction between the oxidized/reduced species and the electrolyte thanks to the close contacts established in this kind of core@shell heterostructure between the Au core and the PBA shell. This fact is corroborated by comparing these results with the lower current densities obtained in systems where the contacts between the two components are much weaker (PBA NPs decorated with Au NPs, Au-PBA) or inexistent (physical mixture of both NPs, Au+PBA) (see Figures S10, S11 and S14). These compounds display ECSA values considerably higher than that of the PBA NPs because Au exhibits a huge ECSA by itself (value of $0.18 \mu \mathrm{F} \cdot \mathrm{cm}^{-2}$ ) when 
it is exposed to the electrolyte. Thus, the larger and activated PBA sites from core@ shell heterostructure could be exploited in order to increase the number of sites contributing to electrocatalytic reactions such as the OER.

\subsection{Electrocatalytical performance}

Electrocatalytic activities of different NPs — with and without $\mathrm{Au}$ - were tested and compared by Linear Sweep Voltammetry (LSV) performed now at a slow scan rate in order to minimize capacitive currents (see Figures 3A and 4A). Figures 3B and 4B display a zoom of the starting catalytic region to evidence the electrocatalytic differences. It is important to remark that these curves were not corrected with the solution resistance $(i R$, with $\mathrm{R}=3 \pm 1 \Omega$ ) to make a fair comparison of the intrinsic electrocatalytic behavior of each sample. When we compare the polarization curves from the different PBA NPs of Fe ${ }^{\mathrm{II}}$, we observe that Au@PBA materials (both with thick and thin shells) display lower onset potentials, which are lower when the coating shell is thicker. The attained values are even better than those shown by the pristine PBA samples with many defects ( $\mathrm{NiFe}$ and $\mathrm{CoFe})$. On the other hand, both, Au-PBA and $\mathrm{Au}+\mathrm{PBA}$, do not result advantageous in terms of electrocatalysis. In these materials, $\mathrm{Au}$ is exposed to the electrolyte and their LSV resemble the one recorded for Au showing high oxidation overpotentials. In addition, it is important to remind at this point that in the Au-PBA case, a polymer is used to connect both systems. Then, the polymer matrix surrounding Au NPs decreases the conductivity of the heterostructure leading to a lower electrocatalytic performance compared to that shown by the physical mixture Au+PBA.

To analyze the electrocatalytic behavior, some key parameters were compared from the experimental voltammetric responses, namely: (1) overpotential required to get $10 \mathrm{~mA} \cdot \mathrm{cm}^{-2}$ (horizontal dotted lines, Figures 3A and 4A), (2) flowing current density at a constant overpotential of $350 \mathrm{mV}$ (vertical dotted lines, Figures 3B and 4B) and (3) Tafel slopes. The overpotential required at $10 \mathrm{~mA} \cdot \mathrm{cm}^{-2}$ was chosen because it is the approximate current density 
expected for a $10 \%$ efficient solar-to-fuels conversion device under 1 sun illumination. ${ }^{[47]}$ Figures $3 \mathrm{C}$ and $4 \mathrm{C}$ evidence that a thin shell leads to lower overpotentials, with a further decrease when the shell thickness is increased. Indeed, this parameter is reduced from $465 \mathrm{mV}$ to 426 and then to $375 \mathrm{mV}$ for NiFe ${ }^{\mathrm{II}}$, Au@NiFe(thin) and Au@NiFe(thick), respectively. For CoFe ${ }^{\mathrm{II}}, \mathrm{Au} @ \mathrm{CoFe}$ (thin) and Au@CoFe(thick), the overpotential decreases from $405 \mathrm{mV}$ to 380 and then to $357 \mathrm{mV}$, respectively. A similar trend was observed for the current densities flowing at a fixed overpotential of $350 \mathrm{mV}$ (Figures 3D and 4D): for $\mathrm{Au} @ \mathrm{NiFe}$ (thick), the current density increases from 1.6 to $5.1 \mathrm{~mA} \cdot \mathrm{cm}^{-2}$ (i.e. an improvement of $320 \%$ ) and for Au@CoFe(thick), from 1.9 to $8.1 \mathrm{~mA} \cdot \mathrm{cm}^{-2}$ (i.e. an improvement of $420 \%$ ). Besides, the results obtained for Au@PBA with a thick shell exceed considerably the performance recorded for a highly defective pristine PBA ( $\mathrm{NiFe}$ and $\mathrm{CoFe}$ ). On the other hand, when $\mathrm{Au}$ is exposed to the electrolyte (Au-PBA and Au+PBA samples), the heterostructure does not seem to be electrocatalytic at these overpotentials (Figures 3A and 4A) even if they display superior ECSA (Figure S15). Therefore, the electrocatalytic enhancement is originated thanks to the presence of a larger number of activated PBA sites as it occurs in a thick PBA layer surrounding Au. Specifically, the introduction of Au in a core@shell structure yields to a reduction of the overpotential up to $100 \mathrm{mV}$ and an increase up to $420 \%$ of the current density recorded at an overpotential of $350 \mathrm{mV}$. 

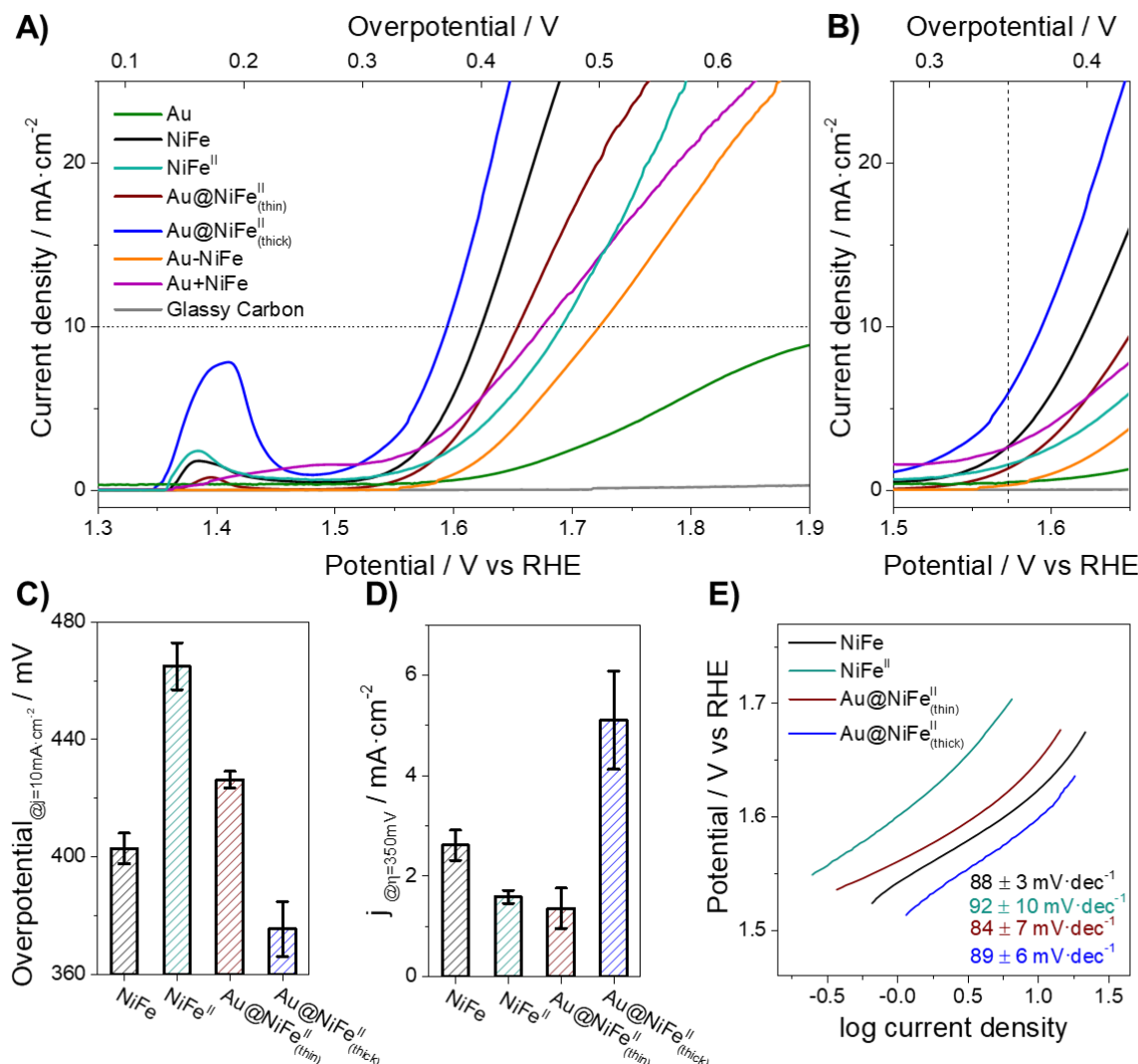

D)

E)
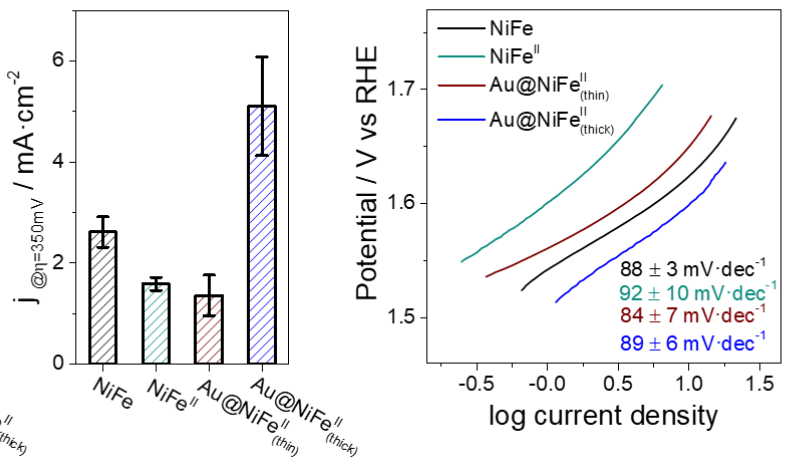

Figure 3. A) Linear Sweep Voltammetry of the different NiFe NPs measured at $5 \mathrm{mV} \cdot \mathrm{s}^{-1}$ in 1 $\mathrm{M} \mathrm{KOH}$ aqueous solution. B) Zoom of Figure 4A exhibiting the beginning of the OER. C) Overpotential required for a current density of $10 \mathrm{~mA} \cdot \mathrm{cm}^{-2}$ for NiFe NPs. D) Current density obtained at an overpotential of $350 \mathrm{mV}$ for NiFe NPs. E) Tafel slopes calculated from LSV data for NiFe NPs. 

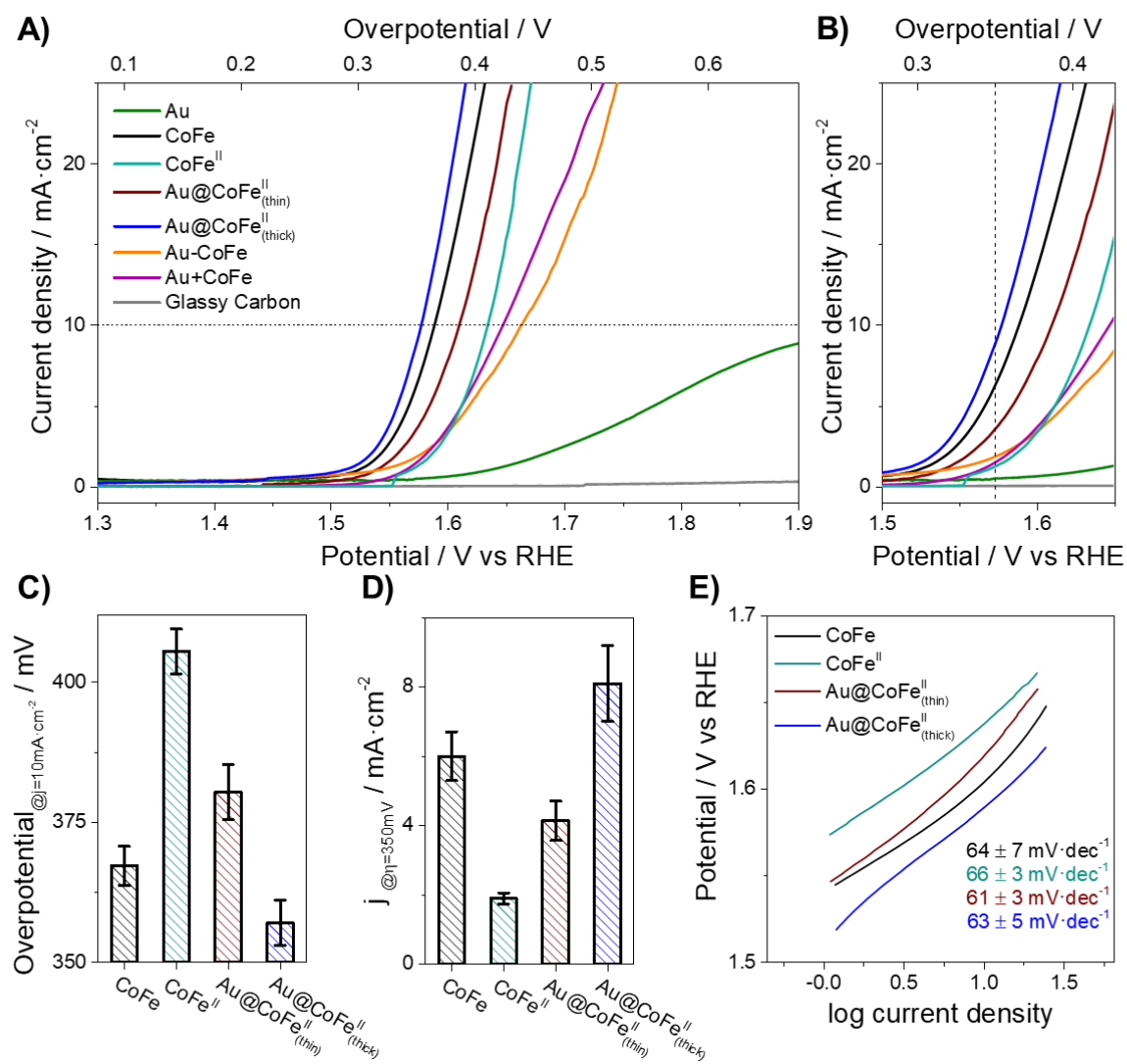

D)

E)
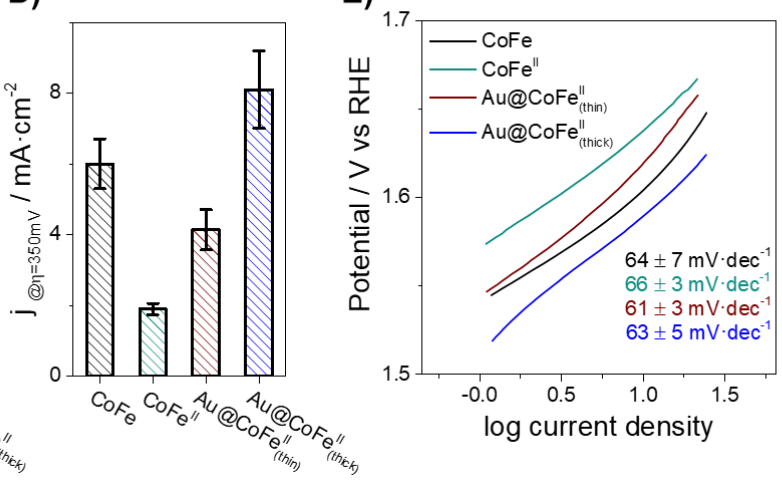

Figure 4. A) Linear Sweep Voltammetry of the different CoFe NPs measured at $5 \mathrm{mV} \cdot \mathrm{s}^{-1}$ in 1 $\mathrm{M} \mathrm{KOH}$ aqueous solution. B) Zoom of Figure 5A exhibiting the beginning of the OER. C) Overpotential required for a current density of $10 \mathrm{~mA} \cdot \mathrm{cm}^{-2}$ for CoFe NPs. D) Current density obtained at an overpotential of $350 \mathrm{mV}$ for CoFe NPs. E) Tafel slopes calculated from LSV data for CoFe NPs.

Regarding the kinetics, Figures 3E and 4E show the Tafel slope values calculated from the LSV responses from $\mathrm{NiFe}$ and $\mathrm{CoFe}$ compounds, respectively. When Au oxidation is not taking place (either in the absence of Au or in the core@ shell structure), similar Tafel slopes are observed, pointing to a small influence on both, the mechanism and kinetics of the oxygen release. Thus, the addition of Au decreases significantly the onset potential but it does not affect the Tafel slope, indicating that $\mathrm{Au}$ reduces the reaction overpotential without affecting the reaction kinetics. These results are in good agreement with those reported for other core@shell structures based on an Au core ${ }^{[16,48]}$ supporting that this kind of nanostructure has a strong synergistic effect between the core and the shell. Trying to corroborate this point, 
Electrochemical Impedance Spectroscopy (EIS) measurements were performed (Figures S16A and S16B).

The equivalent circuits used to fit the EIS data are shown in Figures S16C and S16D. Constant phase elements (CPEs), that are non-ideal capacitances, were introduced to provide a good match with the experimental data because of the possible surface roughness, physical nonuniformity and the non-uniform distribution of the electroactive sites. When PBA is the only species interacting with the electrolyte, the equivalent circuit is composed of a resistance coming from the ionic transport through the solution and the current collectors $\left(\mathrm{R}_{\mathrm{s}}\right)$ connected in series with a first parallel branch $\left(\mathrm{R}_{\text {int }}\right.$ and $\left.\mathrm{CPE}_{\text {int }}\right)$ corresponding to the interfacial contact between the NPs and the Glassy Carbon. These three elements are observed in the highfrequency region. In the low-frequency region, OER processes occurring on the PBA surface are represented by a second parallel branch $\left(\mathrm{R}_{\mathrm{PBA}}\right.$ and $\left.\mathrm{CPE}_{\mathrm{PBA}}\right)$. As observed in Figure 5, $\mathrm{R}_{\mathrm{PBA}}$ is significantly decreased due to the existence of defects in the PBA NPs, but an extraordinary drop is observed for the Au@NiFe heterostructures, leading to an enhancement of the electrocatalytic properties. As expected, a thinner PBA shell gives rise to lower resistance. Still, the incorporation of 5-10\% of $\mathrm{Au}$ is enough to reduce this resistance by 4 for NiFe and by 3 in CoFe. 

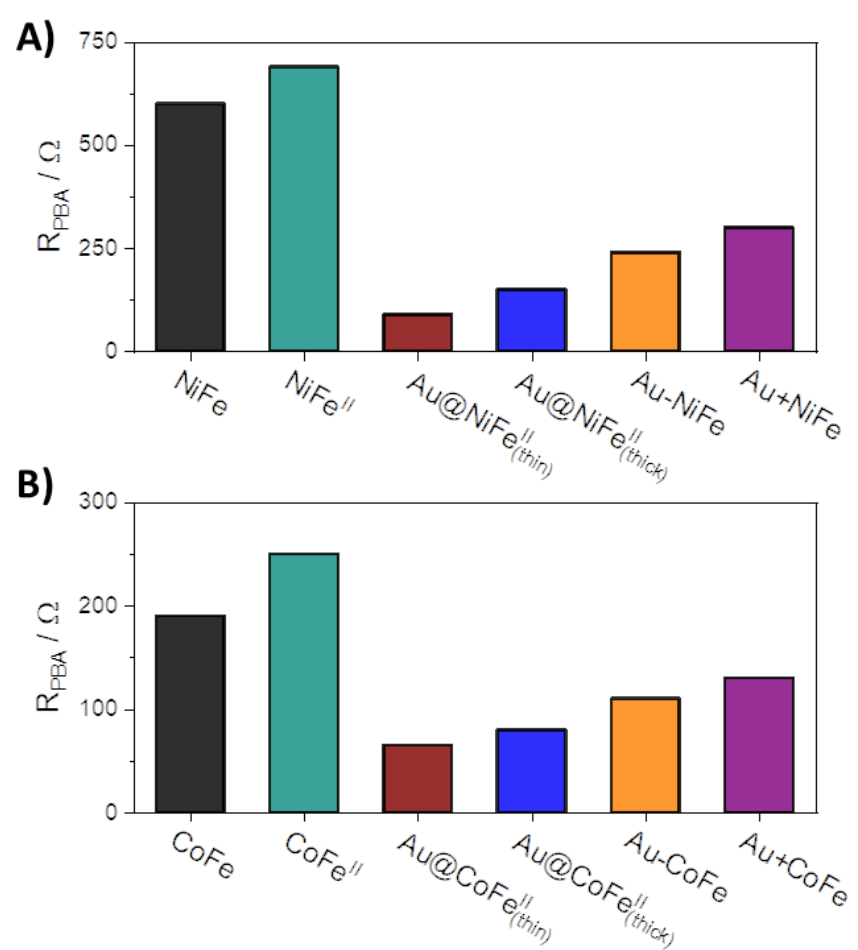

Figure 5. Resistance values of the PBA associated with the OER process for $\mathrm{A}$ ) $\mathrm{NiFe}$ compounds and B) $\mathrm{CoFe}$ compounds. These resistance values were calculated from the equivalent circuit shown in Figure S16.

Summarizing, by comparing the different systems exhibiting the same Au loading (i.e. Au@PBA(thin), Au-PBA and Au+PBA), one can conclude that the appropriate design of the heterostructure is crucial in order to maximize the electrocatalytic activity. In fact, Au activates the PBA sites and increases the conductivity of the hybrid but must be protected to prevent its oxidation. In this case, the Au@PBA system turns out to be the most suitable nanoarchitecture. Here, a thick PBA layer further improves the catalytic performance thanks to the larger number of activated PBA sites acting on the OER and the still important increment in conductivity. Compared with previously reported OER electrocatalysts where their electrical conductivity was improved following different strategies to decrease the onset potential, our strategy turns out to be very effective (Table 1). Indeed, the introduction of small amounts of Au in a core@ shell structure gives rise to a higher reduction of the voltage than using reduced graphene oxide (rGO). In addition to this, the overpotentials at $10 \mathrm{~mA} \cdot \mathrm{cm}^{-2}$ match the values obtained 
for $\mathrm{IrO}_{2}$ and are comparable to other reported electrocatalysts (Table S4). Furthermore, our electrocatalysts still display room for improvement since the electrochemical activity can be further improved by using other supporting electrodes (such as Ni foam) and by subjecting the heterostructures to different thermal and physical pretreatments (such as plasma activation).

Table 1. Comparison of overpotential improvement for the OER using previously reported strategies to increase the electrical conductivity of the electrocatalyst.

\begin{tabular}{c|c|c|c}
\multicolumn{2}{c|}{ Sample comparison } & $\begin{array}{c}\text { Overpotential decrease } \\
\text { at 10 mA } \mathbf{c m}^{-2}(\mathbf{m V})\end{array}$ & Reference \\
\hline $\mathrm{Au} @ \mathrm{NiFe}$ & $\mathrm{NiFe}$ & 92 & This work \\
$\mathrm{Au} @ \mathrm{CoFe}$ & $\mathrm{CoFe}$ & 51 & This work \\
$\mathrm{Au} @ \mathrm{CoO}_{4}$ & $\mathrm{CoO}_{4}$ & 50 & {$[16]$} \\
$\mathrm{ZnCo} \mathrm{O}_{4} / \mathrm{Au} / \mathrm{CNTs}$ & $\mathrm{ZnCo}_{2} \mathrm{O}_{4} / \mathrm{CNTs}$ & 31 & {$[46]$} \\
$\mathrm{Au} @ \mathrm{NiO}$ & $\mathrm{NiO}$ & 90 & {$[48]$} \\
$\mathrm{Au} @ \mathrm{CoFeO}$ & $\mathrm{CoFeO}$ & 39 & {$[48]$} \\
$\mathrm{CNTs}-\mathrm{Au} @ \mathrm{Co}_{3} \mathrm{O}_{4}$ & $\mathrm{CNTs} @ \mathrm{Co}_{3} \mathrm{O}_{4}$ & 50 & {$[49]$} \\
$\mathrm{Au} / \mathrm{NiFe}-\mathrm{LDH}$ & $\mathrm{NiFe}-\mathrm{LDH}$ & 30 & {$[50]$} \\
$\mathrm{Ag}+\mathrm{Co}(\mathrm{OH})_{2}$ & $\mathrm{Co}(\mathrm{OH})_{2}$ & 30 & {$[51]$} \\
$\mathrm{Ag} @ \mathrm{Co}(\mathrm{OH})_{2}$ & $\mathrm{Co}(\mathrm{OH})_{2}$ & 100 & {$[51]$} \\
$\mathrm{FeNi}-\mathrm{rGO}$ & $\mathrm{FeNi}$ & 30 & {$[52]$} \\
$\mathrm{NiO}-\mathrm{NiFe} \mathrm{O}_{4} / \mathrm{rGO}$ & $\mathrm{NiO}-\mathrm{NiFe} \mathrm{O}_{4}$ & 75 & {$[53]$} \\
$\mathrm{Co}(\mathrm{OH})_{2} / \mathrm{SWNT}$ & $\mathrm{Co}(\mathrm{OH})_{2}$ & 40 & {$[54]$} \\
$\mathrm{Ni}-\mathrm{Fe}-\mathrm{Y}-\mathrm{O}_{\mathrm{x}}-\mathrm{Ni}$ foam & $\mathrm{Ni}-\mathrm{Fe}-\mathrm{Y}-\mathrm{O}_{\mathrm{x}}-\mathrm{GC}$ & 27 & {$[55]$}
\end{tabular}

\subsection{Electrochemical stability}

Last but not least, the long-term stability of $\mathrm{NiFe}^{\mathrm{II}}$ and $\mathrm{CoFe}^{\mathrm{II}}$ with and without $\mathrm{Au}$ were compared for 24 hours under oxygen evolution at a constant current density of $20 \mathrm{~mA} \cdot \mathrm{cm}^{-2}$ (Figure 6). Note that Ni foam was used to avoid the detachment of the electrocatalyst from the electrode surface during the $\mathrm{O}_{2}$ formation. The reaction overpotentials are lower than those expected from Figures 3 and 4 for the same current density, due to the porous structure of the substrate used to support the NPs in these measurements (Ni foam foil). ${ }^{[5]}$ The best electrocatalytic stability was observed when introducing 5-10\% in weight of Au. Thus, after 
24 hours of OER, the overpotential increment is $50 \%$ lower in the core@ shell NPs than when the $\mathrm{Au}$ is absent. It seems that $\mathrm{Au}$ gives rise to lower overpotentials which favors the long time stability of the PBA during the OER. It is worth noting that Au-PBA nanostructures exposing $\mathrm{Au}$ to the electrolyte exhibit a progressive voltage increase caused by $\mathrm{Au}$ oxidation and dissolution.

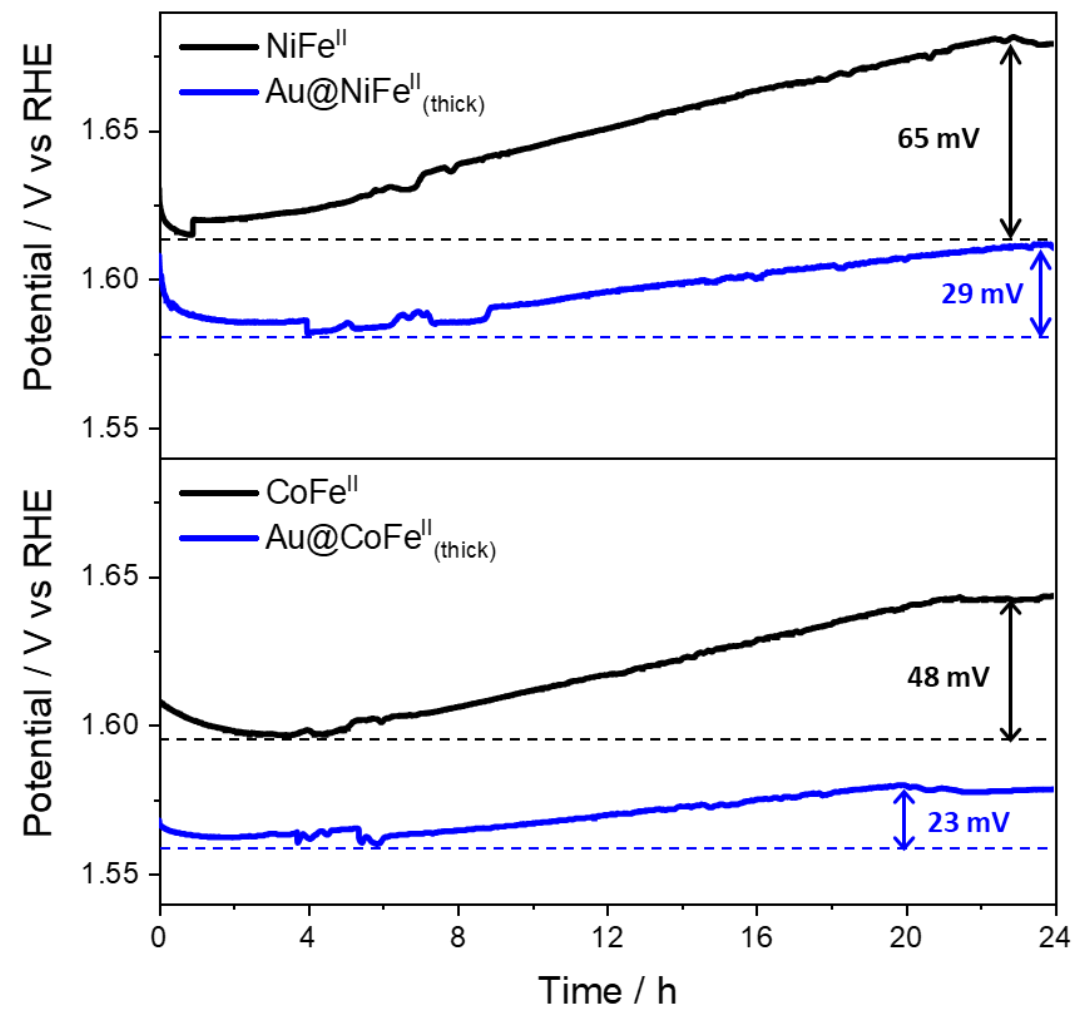

Figure 6. Stability of $\mathrm{NiFe}^{\mathrm{II}}$ and $\mathrm{CoFe}^{\mathrm{II}}$ with and without $\mathrm{Au}$, under a constant current density of $20 \mathrm{~mA} \cdot \mathrm{cm}^{-2}$ for $24 \mathrm{~h}$.

Coulovoltametric responses before and after the stability test can be analyzed to calculate the electroactive mass involved in the redox processes (including $\mathrm{Ni}$ foam). A reduction of the electroactive mass of around $32 \%$ for $\mathrm{NiFe}^{\mathrm{II}}$ but only $20 \%$ for the $\mathrm{Au} @ \mathrm{NiFe}$ (thick) sample was found (Figure S17). For CoFe these values are $25 \%$ and $15 \%$, respectively (Figure S18). The increment of the reaction overpotential is likely a consequence of the loss of electroactive mass caused by the physical detachment or the progressive inactivity of these electroactive centers. Accordingly, these results prove that a proper Au incorporation greatly enhances the 
electrochemical activity of the electroactive PBA shell, improving at the same time its electrochemical stability. Along this front, a core@shell heterostructure with a good Au coverage is necessary to prevent its oxidation.

\section{Conclusion}

We have reported here a variety of narrow-size distributed core@ shell NPs of Au@PBA (PBA $=$ Prussian Blue Analogues of $\mathrm{Ni}^{\mathrm{II}} \mathrm{Fe}^{\mathrm{II}}$ and $\mathrm{Co}^{\mathrm{II}} \mathrm{Fe}^{\mathrm{II}}$ ) formed by an Au core and a PBA shell of different thicknesses. The electrocatalytical activity of such NPs has been evaluated and compared to some PBA and other Au-PBA nanostructures. Compared to the PBA without Au, it was found that the introduction of small amounts of $\mathrm{Au}$ (5-10\% in weight) in the core@ shell structure gives rise to a reduction of the overpotentials at $10 \mathrm{~mA} \cdot \mathrm{cm}^{-2}$ up to $100 \mathrm{mV}$ and an increase up to $420 \%$ of the current density recorded at an overpotential of $350 \mathrm{mV}$ compared to the PBA without Au. Moreover, these voltage decreases are higher than the ones using reduced graphene oxide. Additionally, the Tafel slope remains unaffected indicating that $\mathrm{Au}$ reduces the limiting potential of the catalyst with no variation in the kinetics of the reaction. It was detected for these heterostructures an important increment in the electroactive mass able to be reduced or oxidized and thus, being able to participate in the OER. This in combination with the higher conductivity of the hybrid leads to an enhancement of the electrocatalytic activity that improves at the same time its electrochemical stability. These effects are not observed in the other Au-PBA nanostructures mainly due to the lower contact between both compounds and the oxidation of Au. Therefore, a core@ shell heterostructure with a good Au coverage is required in order to get a protective and electroactive layer of PBA. These improvements are a consequence of the strong synergistic effect between the core and the shell of the Au@PBA nanostructures, which is facilitated by the close contact between both components. This work illustrates the importance of the chemical design for preparing PBA-based nanostructures 
exhibiting better electrocatalytic performances and higher electrochemical stabilities. In subsequent studies both, the electrochemical activity and stability of these materials will be further improved by different thermal and physical pretreatments. This will be facilitated, for example, by exploiting the plasmonic properties of $\mathrm{Au}$, which will allow us to induce a photothermal effect in these nanostructures.

\section{Experimental Section}

Materials:

All chemical reagents were purchased and used without further purification. Potassium ferricyanide, gold (I) potassium cyanide, nickel (II) chloride hexahydrate, cobalt (II) chloride hexahydrate, potassium borohydride, Chloroauric acid, sodium citrate tribasic dehydrate, thiol polyethyleneglycole amine (HS-PEG $3.5 \mathrm{~K}-\mathrm{NH}_{2}$ ), potassium hydroxide $(99.99 \%)$ and Nafion (117 solution) were purchased from Sigma-Aldrich. Carbon black, acetylene 50\% compressed, was obtained from Alfa Aesar (99.9\%). Milli-Q water was obtained from a Millipore Milli-Q equipment.

\section{Nanoparticles synthesis}

To prepare Au@ NiFe NPs, 0.20 mmol of potassium borohydride $\left(\mathrm{KBH}_{4}\right)$ was added to $100 \mathrm{~mL}$ of an aqueous $0.5 \mathrm{mM}$ solution of $\mathrm{K}\left[\mathrm{Au}(\mathrm{CN})_{2}\right]$ under vigorous stirring at around $10{ }^{\circ} \mathrm{C}$. After 20-30 min, aqueous solutions of $\mathrm{K}_{3}\left[\mathrm{Fe}(\mathrm{CN})_{6}\right](5.7 \mathrm{mM})$ and $\mathrm{NiCl}_{2} \cdot 6 \mathrm{H}_{2} \mathrm{O}(5.0 \mathrm{mM})$ were added simultaneously at a rate of $2 \mathrm{~mL} \cdot \mathrm{h}^{-1}$ to the Au NPs solution under vigorous stirring. After completion of the addition, the solution was vigorously stirred for half an hour. In order to prepare Au@CoFe NPs, $0.20 \mathrm{mmol}$ of $\mathrm{KBH}_{4}$ was added to $100 \mathrm{~mL}$ of an aqueous $0.5 \mathrm{mM}$ solution of $\mathrm{K}\left[\mathrm{Au}(\mathrm{CN})_{2}\right]$ under vigorous stirring at around $10{ }^{\circ} \mathrm{C} .10-15$ minutes after the solution turns red, aqueous solutions of $\mathrm{K}_{3}\left[\mathrm{Fe}(\mathrm{CN})_{6}\right](5.7 \mathrm{mM})$ and $\mathrm{CoCl}_{2} \cdot 6 \mathrm{H}_{2} \mathrm{O}(5.0 \mathrm{mM})$ were 
added simultaneously at an addition rate of $0.5 \mathrm{~mL} \cdot \mathrm{h}^{-1}$ to the Au NPs solution under vigorous stirring. After completion of the addition, the solution was vigorously stirred for half an hour. Core@ shell NPs were washed with water (11000 rpm for 20 minutes) and finally were dried under vacuum. A thinner and a thicker shell were achieved by adding respectively 2 and $10 \mathrm{~mL}$ of each precursor solution. $\mathrm{K}\left[\mathrm{Au}(\mathrm{CN})_{2}\right]$ reduction was carried out in an ice bath in order to produce smaller Au cores.

PBA-NiFe NPs of around $150 \mathrm{~nm}$ were synthesized at room temperature by adding, to $100 \mathrm{~mL}$ aqueous solution at $2 \mathrm{~mL} \cdot \mathrm{h}^{-1}$ rate, aqueous solutions of $\mathrm{CoCl}_{2} \cdot 6 \mathrm{H}_{2} \mathrm{O}(5.0 \mathrm{mM}, 7 \mathrm{~mL})$ and $\mathrm{K}_{3}\left[\mathrm{Fe}(\mathrm{CN})_{6}\right](5.7 \mathrm{mM}, 7 \mathrm{~mL})$ were added simultaneously. PBA-CoFe NPs of around $180 \mathrm{~nm}$ were also synthesized at room temperature by adding, to $100 \mathrm{~mL}$ aqueous solution at $2 \mathrm{~mL} \cdot \mathrm{h}^{-1}$ rate, aqueous solutions of $\mathrm{NiCl}_{2} \cdot 6 \mathrm{H}_{2} \mathrm{O}(5.0 \mathrm{mM}, 8 \mathrm{~mL})$ and $\mathrm{K}_{3}\left[\mathrm{Fe}(\mathrm{CN})_{6}\right](5.7 \mathrm{mM}, 8 \mathrm{~mL})$. After completion of the addition, the mixtures were stirred for half an hour before being centrifuged at $11000 \mathrm{rpm}$ for $20 \mathrm{~min}$. The supernatants were removed, and the powders were let dried under vacuum. PBA-NiFe ${ }^{I I}$ and PBA-CoFe ${ }^{I I}$ were prepared using the same synthetic procedure but adding $0.4 \mathrm{mmol}$ of potassium borohydride $\left(\mathrm{KBH}_{4}\right)$ to promote the reduction of $\mathrm{Fe}^{\mathrm{III}}$.

$\mathrm{Au}$ NPs stabilized by citrate capping agent were synthesized following the well-known Turkevich method. ${ }^{[57]}$ Physical mixture (Au+PBA) was prepared by joining in weight $30 \%$ of Au NPs and $70 \%$ of PBA NPs.

The decoration of Au on PBA NPs was carried out by connecting each NP by a polymer containing a thiol and an amine group(HS-PEG-NH 2 ) following a protocol developed in our group. ${ }^{[39]}$

\section{Electrode preparation}

For the electrode preparation, a dispersion composed of $1 \mathrm{mg}$ of powder material, $0.5 \mathrm{mg}$ of acetylene black, $200 \mu \mathrm{L}$ of water and ethanol (1:1) and $8 \mu \mathrm{L}$ of Nafion (10\%) was sonicated in order to obtain a well-dispersed suspension. Then, 3.6 $\mu \mathrm{L}$ was drop-casted in a previously 
polished (sequentially with $1.0,0.3$ and $0.05 \mu \mathrm{m}$ alumina powder) $3 \mathrm{~mm}$ Glassy Carbon electrode. Afterwards, the solvent was let evaporated at room temperature. The electrode mass loading achieved was around $0.25 \mathrm{mg} \cdot \mathrm{cm}^{-2}$.

\section{Electrochemical measurements}

Electrochemical tests were performed in a typical three-electrode cell equipped with Glassy Carbon acting as the working electrode and a platinum wire as the counter electrode. As the reference electrode, a silver-silver chloride (versus $\mathrm{Ag} / \mathrm{AgCl}(3 \mathrm{M} \mathrm{KCl})$ ) was used. All potentials were converted referring to the oxygen evolution overpotential or the reversible hydrogen electrode (RHE). The measurements were performed at least three times for every sample using different electrodes on an Autolab PGSTAT 128N potentiostat/galvanostat. Linear sweep voltammetry (LSV) measurements were carried out at $5 \mathrm{mV} \cdot \mathrm{s}^{-1}$ in a previously $\mathrm{N}_{2}$ purged $1 \mathrm{M} \mathrm{KOH}$ aqueous solution. Prior to this, cyclic voltammetries (CVs) were performed at different scan rates $\left(100,50,20\right.$ and $\left.10 \mathrm{mV} \cdot \mathrm{s}^{-1}\right)$.

Electrochemical surface area (ECSA) was acquired by measuring the current associated with double-layer capacitance from the scan rate dependence of CVs. The potential range used for the CVs was from -0.2 to $0.1 \mathrm{~V}$ versus $\mathrm{Ag} / \mathrm{AgCl}(3 \mathrm{M} \mathrm{KCl})$. The scan rates were 400, 300, 200, 100 and $50 \mathrm{mVs}^{-1}$. The double layer capacitance was estimated by plotting the (ja-jc) (anodic versus cathodic currents) at $-0.05 \mathrm{~V}$ versus $\mathrm{Ag} / \mathrm{AgCl}(3 \mathrm{M} \mathrm{KCl})$ against the scan rate. The ECSA was measured on the working electrodes after performing an activation process consisting of 5 $\mathrm{CVs}$ at $50 \mathrm{mV} \cdot \mathrm{s}^{-1}$ around their redox processes.

Electrochemical impedance spectroscopy (EIS) measurements were carried out using a Gamry 1000E potentiostat/galvanostat controlled by Gamry software by applying an AC amplitude of $10 \mathrm{mV}$ in the frequency range of $10^{-1}-10^{5} \mathrm{~Hz}$ at an overpotential of $0.4 \mathrm{~V}$. EIS data were analyzed and fitted by means of Gamry Echem Analyst v. 7.07 software. 
Stability tests were performed under a constant current density of $20 \mathrm{~mA} \cdot \mathrm{cm}^{-2}$ during $24 \mathrm{~h}$ using $\mathrm{Ni}$ foam foil (which area is $0.6 \mathrm{~cm}^{2}$ ) as the working electrode containing $0.25 \mathrm{mg} \cdot \mathrm{cm}^{-2}$ of electrocatalyst mass.

\section{Physical characterization}

UV/Vis Spectroscopy: UV-vis absorption spectra were recorded on a Jasco V-670 spectrophotometer in baseline mode from 300 to $900 \mathrm{~nm}$ range, using 1.000-cm-optical-path plastic cuvettes.

Inductively Coupled-Plasma Optical Emission Spectrometry (ICP-OES): The ICP-OES analysis were conducted at the Universidad de Valencia (Sección de Espectrometría Atómica y Molecular). Samples were digested in an acid medium at $220{ }^{\circ} \mathrm{C}$ using a microwave oven.

Transmission Electron Microscopy (TEM): TEM studies were carried out on a JEOL JEM 1010 microscope operating at $100 \mathrm{kV}$, and Technai G2 F20 microscope operating at $200 \mathrm{kV}$. Samples were prepared by dropping suspensions on lacey formvar/carbon copper grids (300 mesh).

Magnetic Measurements: Magnetic data were collected with a Quantum Design MPMS XL-5 susceptometer equipped with a SQUID sensor. Field Cooling magnetization measurements were performed under a magnetic field applied of 1000 Oe.

Raman Spectroscopy: Raman spectra were acquired with a Raman Emission Horiba-MTB Xplora Spectrometer in ambient conditions. NPs were measured with a laser wavelength of 532 $\mathrm{nm}$ by drop-casting the samples onto silicon substrates.

X-Ray Powder Diffraction (XRPD): X-ray powder diffraction (XRPD) patterns were obtained with a PANalytical X'Pert diffractometer using the copper radiation $(\mathrm{Cu}-\mathrm{Ka}=1.54178 \AA)$ in the 5-50 region.

X-ray Photoelectron Spectroscopy (XPS): Samples were analyzed using a K-ALPHA Thermo Scientific spectrometer. All spectra were collected using Al Ka radiation (1486.6 eV), monochromatized by a twin crystal monochromator, yielding a focused X-ray spot (elliptical 
in shape with a major axis length of $400 \mu \mathrm{m}$ ) at $30 \mathrm{~mA}$ and $2 \mathrm{kV}$. The alpha hemispherical analyzer was operated in the constant energy mode with survey scan pass energies of $200 \mathrm{eV}$ to measure the whole energy band and $50 \mathrm{eV}$ in a narrow scan to selectively measure the particular elements. XPS data were analyzed with Avantage software. A smart background function was used to approximate the experimental backgrounds. Charge compensation was achieved with the system flood gun that provides low energy electrons and low energy argon ions from a single source.

\section{Acknowledgements}

The authors acknowledge funding from the EU (ERC Advanced Grant Mol-2D 788222, and FET-OPEN COSMICS 766726), the Spanish MCIU (Unit of Excellence "Maria de Maeztu" CEX2019-000919-M and Project MAT2017-89993-R co-financed by FEDER) and the Generalitat Valenciana (Prometeo Programme PROMETEO/2017/066, APOSTD contract to M.C-P and iDiFEDER/2018/061). R.S.-G. thanks the Spanish MCIU for F.P.U. fellowships. The authors thank Prof. Talal Mallah for helpful discussions.

\section{References}

[1] F. Song, L. Bai, A. Moysiadou, S. Lee, C. Hu, L. Liardet, X. Hu, J. Am. Chem. Soc. 2018, 140,7748 .

[2] Z. P. Wu, X. F. Lu, S. Q. Zang, X. W. Lou, Adv. Funct. Mater. 2020, 30, 1.

[3] G. Abellán, J. A. Carrasco, E. Coronado, J. Romero, M. Varela, J. Mater. Chem. C 2014, 2, 3723 .

[4] Z. Shi, X. Wang, J. Ge, C. Liu, W. Xing, Nanoscale 2020, 12, 13249.

[5] F. Lyu, Q. Wang, S. M. Choi, Y. Yin, Small 2019, 15, 1.

[6] M. Verdaguer, G. S. Girolami, Magnetic Prussian Blue Analogues, Vol. 36, 2005.

[7] M. Morant-Giner, R. Sanchis-Gual, J. Romero, A. Alberola, L. García-Cruz, S. Agouram, M. Galbiati, N. M. Padial, J. C. Waerenborgh, C. Martí-Gastaldo, S. Tatay, A. Forment-Aliaga, E. Coronado, Adv. Funct. Mater. 2017, 1706125, 1.

[8] F. Zhao, Y. Wang, X. Xu, Y. Liu, R. Song, G. Lu, Y. Li, ACS Appl. Mater. Interfaces 
2014, 6, 11007.

[9] L. Han, P. Tang, Á. Reyes-Carmona, B. Rodríguez-García, M. Torréns, J. R. Morante, J. Arbiol, J. R. Galan-Mascaros, J. Am. Chem. Soc. 2016, 138, 16037.

[10] L. M. Cao, D. Lu, D. C. Zhong, T. B. Lu, Coord. Chem. Rev. 2020, 407, 213156.

[11] D. Y. Chung, S. Park, P. P. Lopes, V. R. Stamenkovic, Y.-E. Sung, N. M. Markovic, D. Strmcnik, ACS Catal. 2020, 10, 4990.

[12] D. Zhao, Y. Lu, D. Ma, Molecules 2020, 25, 2304.

[13] F. Dionigi, P. Strasser, Adv. Energy Mater. 2016, 6, 1600621.

[14] D. Friebel, M. W. Louie, M. Bajdich, K. E. Sanwald, Y. Cai, A. M. Wise, M. J. Cheng, D. Sokaras, T. C. Weng, R. Alonso-Mori, R. C. Davis, J. R. Bargar, J. K. Nørskov, A. Nilsson, A. T. Bell, J. Am. Chem. Soc. 2015, 137, 1305.

[15] J. G. McAlpin, Y. Surendranath, M. Dincã, T. A. Stich, S. A. Stoian, W. H. Casey, D. G. Nocera, R. D. Britt, J. Am. Chem. Soc. 2010, 132, 6882.

[16] Z. Zhuang, W. Sheng, Y. Yan, Adv. Mater. 2014, 26, 3950.

[17] B. S. Yeo, A. T. Bell, J. Am. Chem. Soc. 2011, 133, 5587.

[18] S. Zou, M. S. Burke, M. G. Kast, J. Fan, N. Danilovic, S. W. Boettcher, Chem. Mater. 2015, 27, 8011.

[19] J. W. D. Ng, M. García-Melchor, M. Bajdich, P. Chakthranont, C. Kirk, A. Vojvodic, T. F. Jaramillo, Nat. Energy 2016, 1, 1.

[20] R. Torres-Cavanillas, R. Sanchis-Gual, J. Dugay, M. Coronado-Puchau, M. GiménezMarqués, E. Coronado, Adv. Mater. 2019, 31, 1900039.

[21] M. B. Gawande, A. Goswami, T. Asefa, H. Guo, A. V. Biradar, D. L. Peng, R. Zboril, R. S. Varma, Chem. Soc. Rev. 2015, 44, 7540.

[22] S. Gong, Y. X. Zhang, Z. Niu, ACS Catal. 2020, 10, 10886.

[23] L. Jing, X. Liang, Z. Deng, S. Feng, X. Li, M. Huang, Biomaterials 2014, 35, 5814.

[24] Y. Yin, Q. Li, S. Ma, H. Liu, B. Dong, J. Yang, D. Liu, Anal. Chem. 2017, 89, 1551. 
[25] J. D. Qiu, H. Z. Peng, R. P. Liang, J. Li, X. H. Xia, Langmuir 2007, 23, 2133.

[26] G. Fu, W. Liu, S. Feng, X. Yue, Chem. Commun. 2012, 48, 11567.

[27] X. Lu, Y. Li, X. Zhang, J. Du, X. Zhou, Z. Xue, X. Liu, Anal. Chim. Acta 2012, 711, 40.

[28] G. Maurin-Pasturel, J. Long, Y. Guari, F. Godiard, M. G. Willinger, C. Guerin, J. Larionova, Angew. Chemie - Int. Ed. 2014, 53, 3872.

[29] G. Maurin-Pasturel, E. Mamontova, M. A. Palacios, J. Long, J. Allouche, J. C. Dupin, Y. Guari, J. Larionova, Dalt. Trans. 2019, 48, 6205.

[30] B. H. Liu, Z. P. Li, J. Power Sources 2009, 187, 527.

[31] J. Liang, C. H. Li, D. R. Talham, Cryst. Growth Des. 2020, 20, 2713.

[32] M. M. Miller, A. A. Lazarides, J. Phys. Chem. B 2005, 109, 21556.

[33] K. M. Mayer, J. H. Hafner, Chem. Rev. 2011, 111, 3828.

[34] C. Wang, O. Ranasingha, S. Natesakhawat, P. R. Ohodnicki, M. Andio, J. P. Lewis, C. Matranga, Nanoscale 2013, 5, 6968.

[35] X. Zhang, Y. L. Chen, R. S. Liu, D. P. Tsai, Reports Prog. Phys. 2013, 76, 046401.

[36] H. J. Buser, A. Ludi, D. Schwarzenbach, W. Petter, Inorg. Chem. 1977, 16, 2704.

[37] S. Juszczyk, C. Johansson, M. Hanson, A. Ratuszna, G. Malecki, J. Phys. Condens. Matter 1994, 6, 5697.

[38] Z. Y. Yu, Y. Duan, J. D. Liu, Y. Chen, X. K. Liu, W. Liu, T. Ma, Y. Li, X. S. Zheng, T. Yao, M. R. Gao, J. F. Zhu, B. J. Ye, S. H. Yu, Nat. Commun. 2019, 10, 1.

[39] R. Sanchis-Gual, I. Susic,a R. Torres-Cavanillas, D. Arenas-Esteban, Sara Bals, T. Mallah, M. Coronado-Puchau, E. Coronado. Submitted.

[40] X. Su, Y. Wang, J. Zhou, S. Gu, J. Li, S. Zhang, J. Am. Chem. Soc. 2018, 140, 11286.

[41] S. Pintado, S. Goberna-Ferrón, E. C. Escudero-Adán, J. R. Galán-Mascarós, J. Am. Chem. Soc. 2013, 135, 13270.

[42] T. F. Otero, M. Alfaro, V. Martinez, M. A. Perez, J. G. Martinez, Adv. Funct. Mater. 
2013, 23, 3929.

[43] T. F. Otero, Electrochim. Acta 2016, 212, 440.

[44] R. Sanchis-Gual, A. Seijas-Da Silva, T. F. Otero, E. Coronado, G. Abellán. Manuscript in Preparation.

[45] J. A. Carrasco, A. Harvey, D. Hanlon, V. Lloret, D. McAteer, R. Sanchis-Gual, A. Hirsch, F. Hauke, G. Abellán, J. N. Coleman, E. Coronado, Chem. Commun. 2019, 55, 3315.

[46] H. Cheng, C. Y. Su, Z. Y. Tan, S. Z. Tai, Z. Q. Liu, J. Power Sources 2017, 357, 1.

[47] C. C. L. Mccrory, S. Jung, I. M. Ferrer, S. M. Chatman, J. C. Peters, T. F. Jaramillo, J. Am. Chem. Soc. 2015, 137, 4347.

[48] A. L. Strickler, M. Escudero-Escribano, T. F. Jaramillo, Nano Lett. 2017, 17, 6040.

[49] Y. Fang, X. Li, Y. Hu, F. Li, X. Lin, M. Tian, X. An, Y. Fu, J. Jin, J. Ma, J. Power Sources 2015, 300, 285.

[50] J. Zhang, J. Liu, L. Xi, Y. Yu, N. Chen, S. Sun, W. Wang, K. M. Lange, B. Zhang, J. Am. Chem. Soc. 2018, 140, 3876.

[51] Z. Zhang, X. Li, C. Zhong, N. Zhao, Y. Deng, X. Han, W. Hu, Angew. Chemie Int. Ed. 2020, 59, 7245 .

[52] X. Long, J. Li, S. Xiao, K. Yan, Z. Wang, H. Chen, S. Yang, Angew. Chemie 2014, 126,7714 .

[53] G. Zhang, Y. Li, Y. Zhou, F. Yang, ChemElectroChem 2016, 3, 1927.

[54] D. McAteer, I. J. Godwin, Z. Ling, A. Harvey, L. He, C. S. Boland, V. Vega-Mayoral, B. Szydłowska, A. A. Rovetta, C. Backes, J. B. Boland, X. Chen, M. E. G. Lyons, J. N. Coleman, Adv. Energy Mater. 2018, $8,1$.

[55] Q. Zhang, N. Liu, J. Guan, ACS Appl. Energy Mater. 2019, 2, 8903.

[56] W. Zheng, M. Liu, L. Y. S. Lee, ACS Energy Lett. 2020, 5, 3260.

[57] J. Turkevich, Gold Bull. 1985, 18, 125. 
[58] F. Song, X. Hu, Nat. Commun. 2014, 5, 1.

[59] C.-H. Chuang, L.-Y. Hsiao, M.-H. Yeh, Y.-C. Wang, S.-C. Chang, L.-D. Tsai, K.-C. Ho, ACS Appl. Energy Mater. 2020. 


\section{Supporting Information}

Enhancing the electrocatalytic activity and stability of Prussian Blue Analogues through the introduction of Au nanoparticles in a core@shell heterostructure

Roger Sanchis-Gual, Toribio F. Otero, Marc Coronado-Puchau, * Eugenio Coronado* 

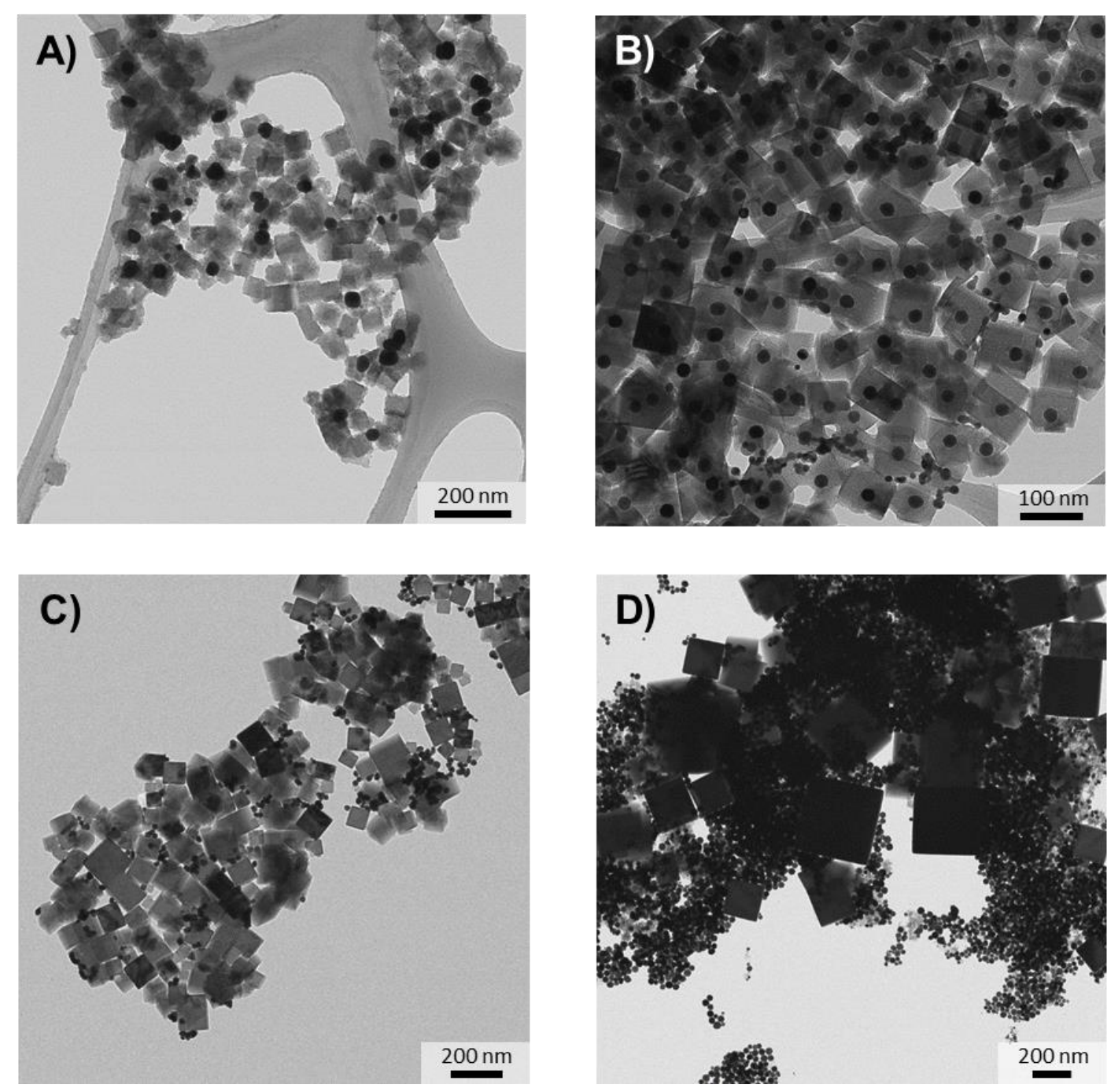

Figure S1. TEM images of the NPs obtained through the Au@CoFe protocol and applying the following parameters: A) addition rate of $2 \mathrm{~mL} \cdot \mathrm{h}^{-1}$ and time delay of 20-30 min. B) addition rate of $0.5 \mathrm{~mL} \cdot \mathrm{h}^{-1}$ and time delay of $20-30 \mathrm{~min}$. C) addition rate of $0.5 \mathrm{~mL} \cdot \mathrm{h}^{-1}$ and time delay of $45 \mathrm{~min}$. D) addition rate of $0.5 \mathrm{~mL} \cdot \mathrm{h}^{-1}$ and time delay of $0 \mathrm{~min}$. 
A)

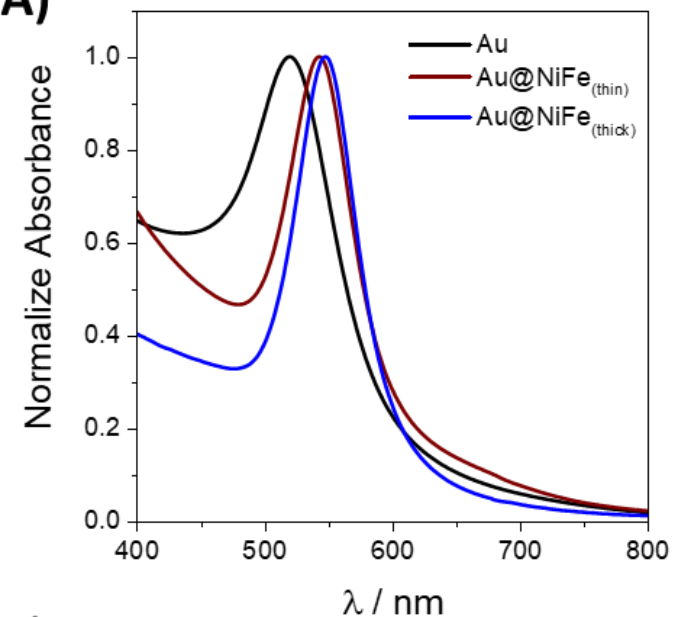

C)

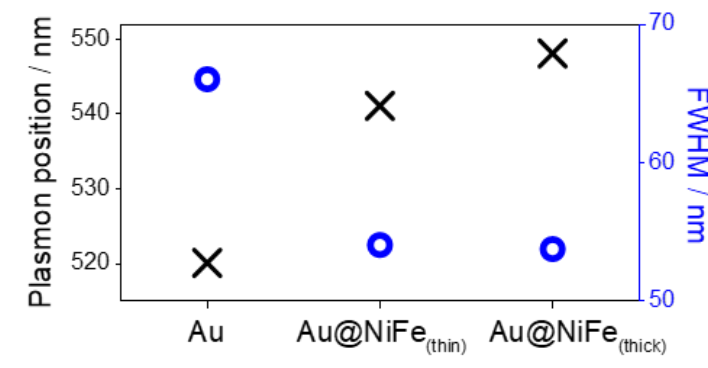

B)

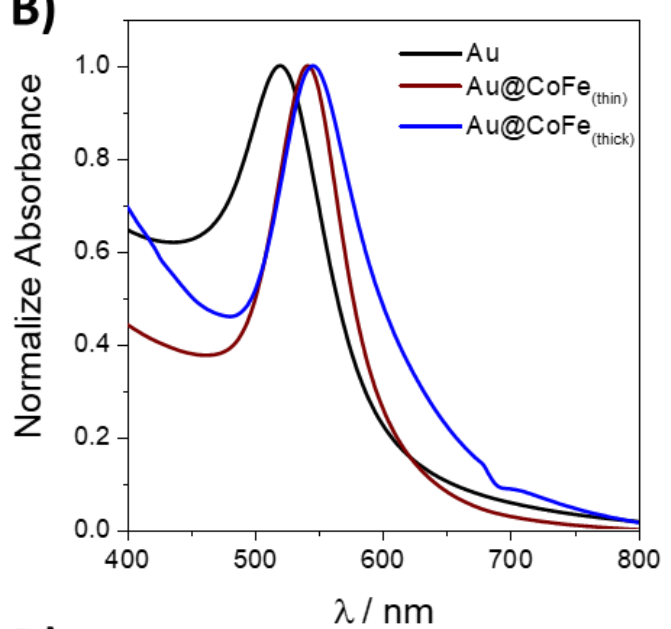

D)

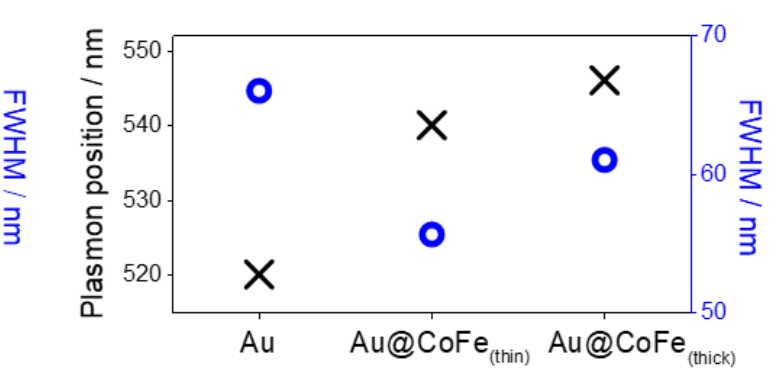

Figure S2. A) B) UV-Vis spectra of Au and the different Au@PBA NPs. C) D) Plasmon position and plasmon FWHM calculated for the different NPs. 

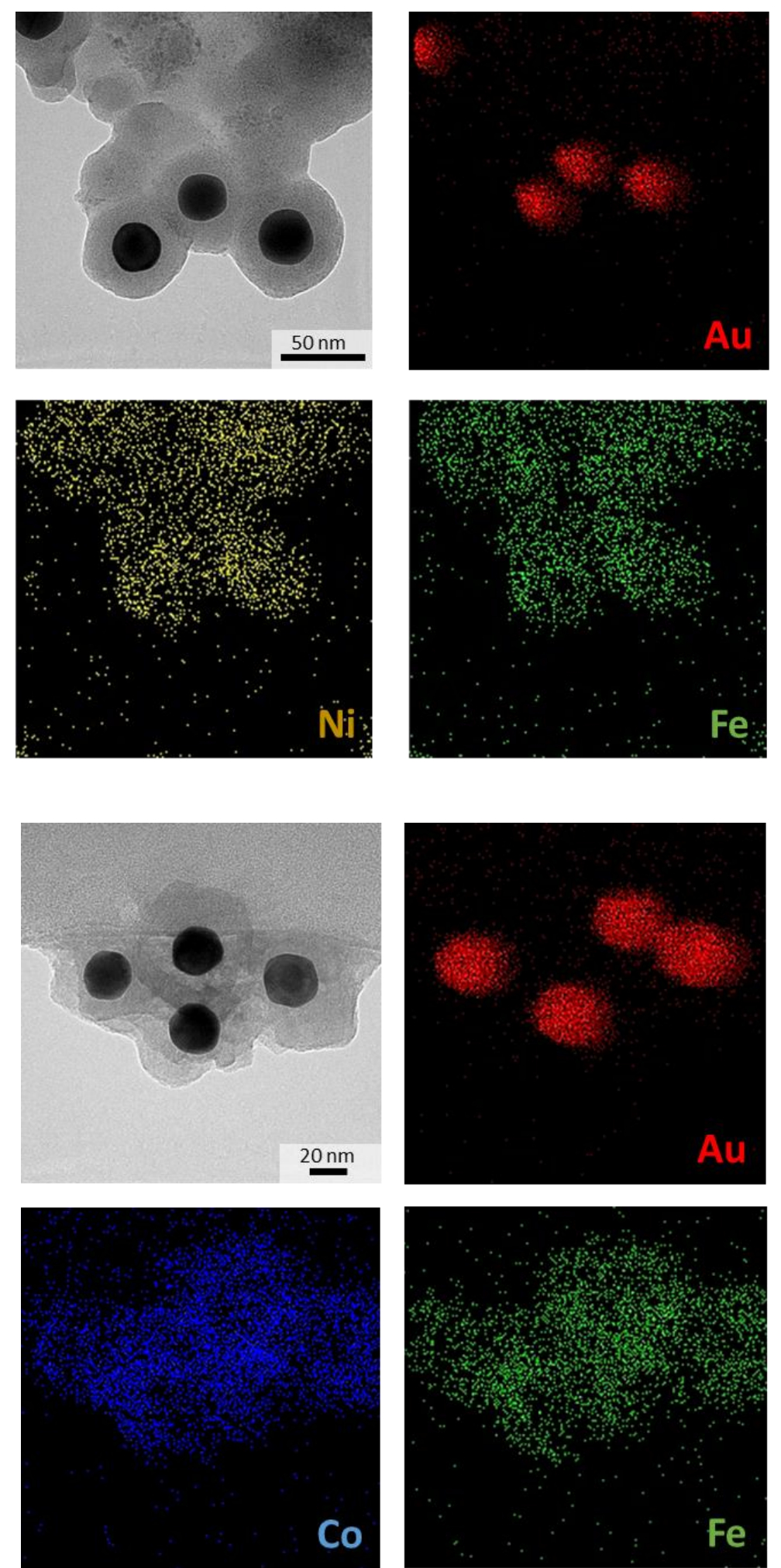

Figure S3. EDX mapping of the metals present in the Au@PBA(thin) heterostructures. 
Table S1. Percentage of gold (in weight) estimated for the different nanoparticles.

\begin{tabular}{l|c} 
Sample & $\boldsymbol{\%} \boldsymbol{A u}(\boldsymbol{w} / \boldsymbol{w})$ \\
\hline $\mathrm{Au} @ \mathrm{NiFe}^{\prime \prime}{ }_{\text {(thin) }}$ & 39 \\
$\mathrm{Au} @ \mathrm{NiFe}^{\prime \prime}{ }_{\text {(thick) }}$ & 9 \\
$\mathrm{Au}-\mathrm{NiFe}$ & 29 \\
$\mathrm{Au+NiFe}$ & 30 \\
$\mathrm{Au} @ \mathrm{CoFe}^{\prime \prime}{ }_{\text {(thin) }}$ & 46 \\
$\mathrm{Au} @ \mathrm{CoFe}{ }^{\prime \prime}{ }_{\text {(thick) }}$ & 6 \\
$\mathrm{Au-CoFe}$ & 31 \\
Au+CoFe & 30
\end{tabular}

Table S2. Molecular formula estimated for the different nanoparticles.

\begin{tabular}{l|l} 
Sample & Molecular formula \\
\hline $\mathrm{Au} @ \mathrm{NiFe}^{\prime \prime}{ }_{\text {(thin) }}$ & $\mathrm{Au}_{0.88} @ \mathrm{~K}_{1.60} \mathrm{Ni}\left[\mathrm{Fe}(\mathrm{CN})_{6}\right]_{0.90}$ \\
$\mathrm{Au} @ \mathrm{NiFe}^{\prime \prime}{ }_{\text {(thick) }}$ & $\mathrm{Au}_{0.13} @ \mathrm{~K}_{1.44} \mathrm{Ni}\left[\mathrm{Fe}(\mathrm{CN})_{6}\right]_{0.86}$ \\
$\mathrm{NiFe}$ & $\mathrm{K}_{0.20} \mathrm{Ni}\left[\mathrm{Fe}(\mathrm{CN})_{6}\right]_{0.72}$ \\
$\mathrm{NiFe}{ }^{\prime \prime}$ & $\mathrm{K}_{1.04} \mathrm{Ni}\left[\mathrm{Fe}(\mathrm{CN})_{6}\right]_{0.76}$ \\
$\mathrm{Au} @ \mathrm{CoFe}^{\prime \prime}{ }_{\text {(thin) }}$ & $\mathrm{Au}_{1.10} @ \mathrm{~K}_{1.52} \mathrm{Co}\left[\mathrm{Fe}(\mathrm{CN})_{6}\right]_{0.88}$ \\
$\mathrm{Au} @ \mathrm{CoFe}^{\prime \prime}{ }_{\text {(thick) }}$ & $\mathrm{Au}_{0.05} @ \mathrm{~K}_{1.56} \mathrm{Co}\left[\mathrm{Fe}(\mathrm{CN})_{6}\right]_{0.89}$ \\
$\mathrm{CoFe}$ & $\mathrm{K}_{0.19} \mathrm{Co}\left[\mathrm{Fe}(\mathrm{CN})_{6}\right]_{0.73}$ \\
$\mathrm{CoFe}{ }^{\prime \prime}$ & $\mathrm{K}_{1.12} \mathrm{Co}\left[\mathrm{Fe}(\mathrm{CN})_{6}\right]_{0.78}$
\end{tabular}



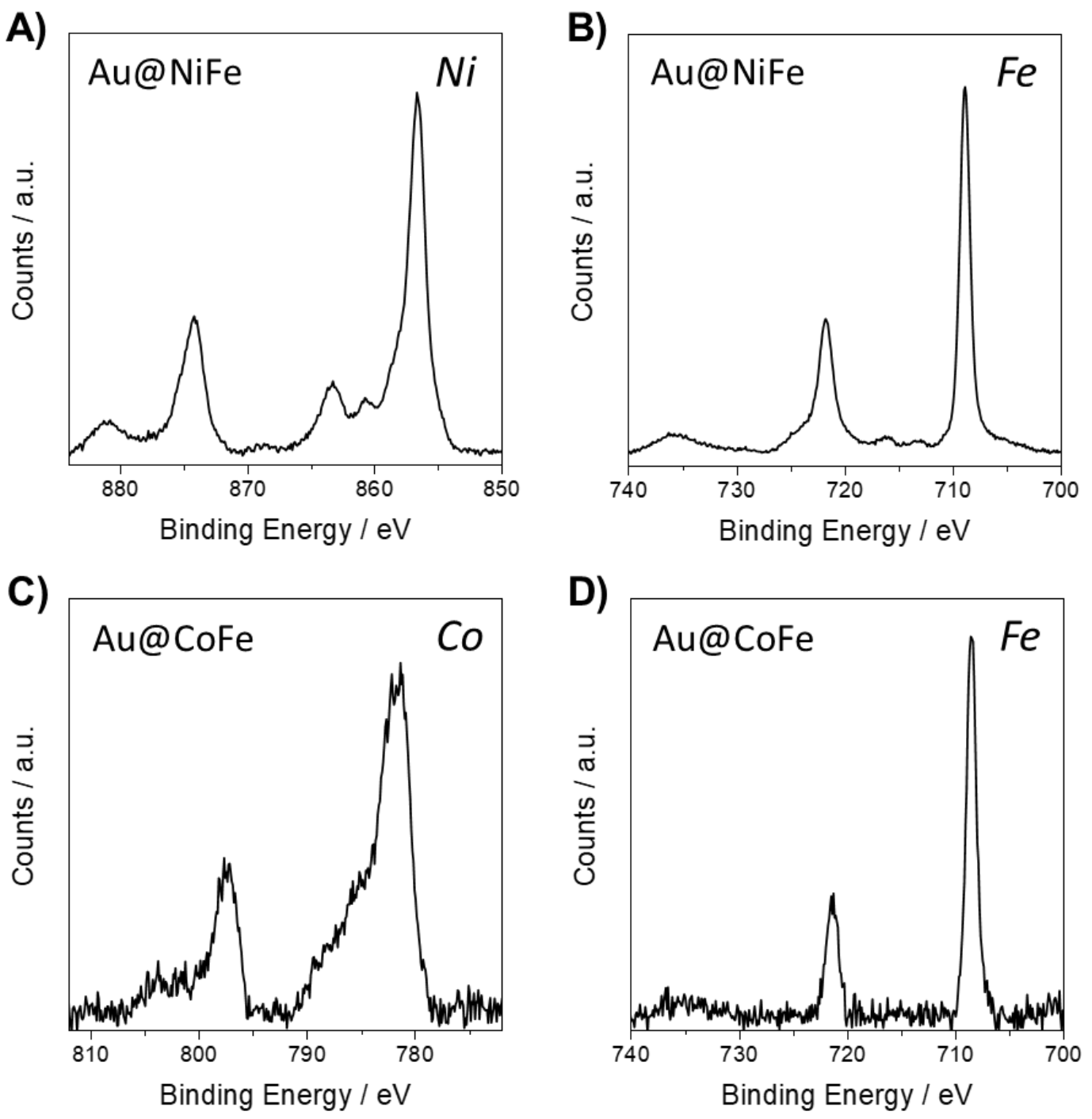

Figure S4. XPS spectra of Au@ NiFe (up) and $\mathrm{Au} @ \mathrm{CoFe}$ (down). 

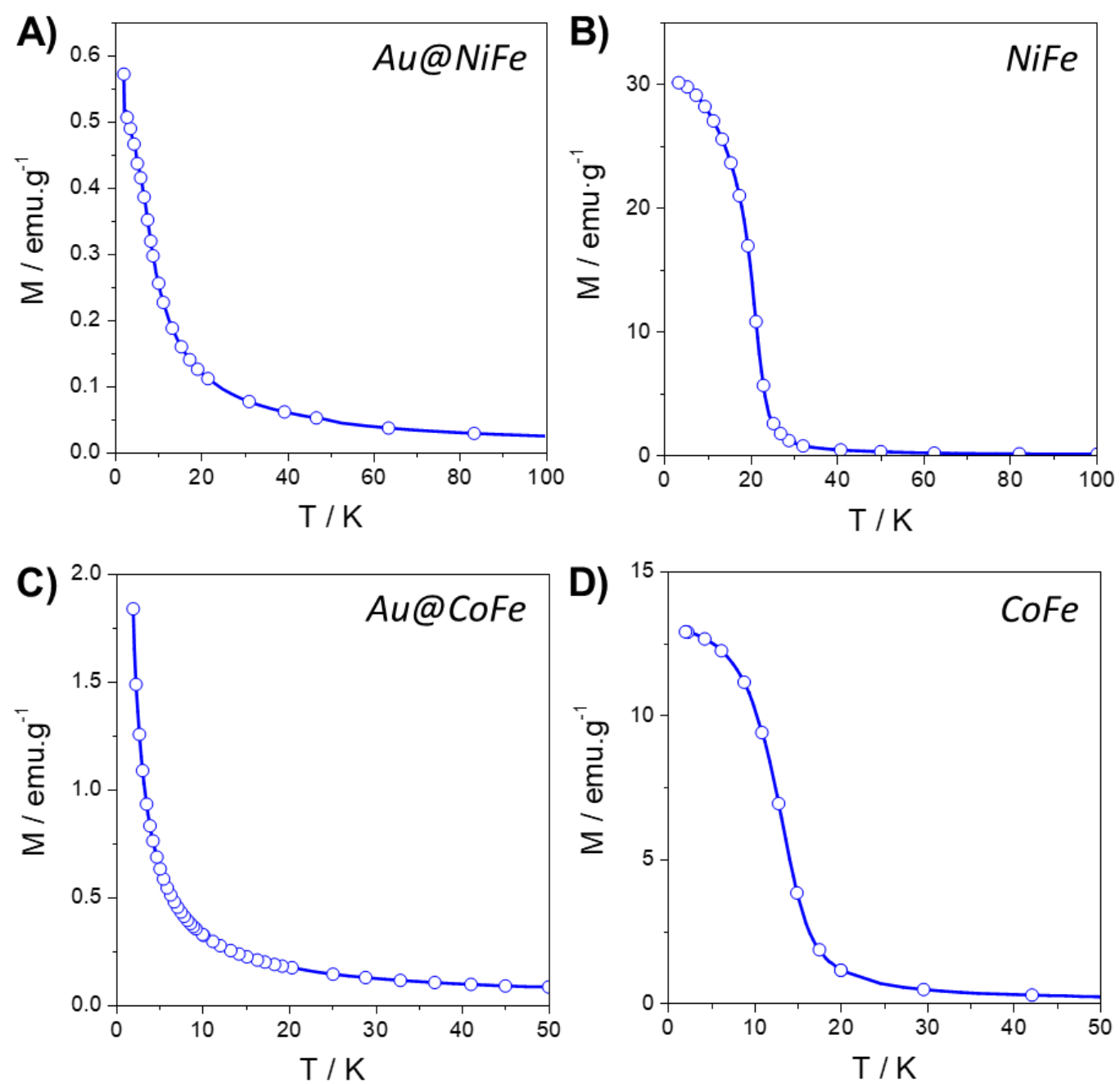

Figure S5. Magnetization vs. temperature curve performed for Au@ NiFe and NIFe-PBA (up) and Au@CoFe and CoFe-PBA (down) nanoparticles with an applied field of 1000 Oe. 

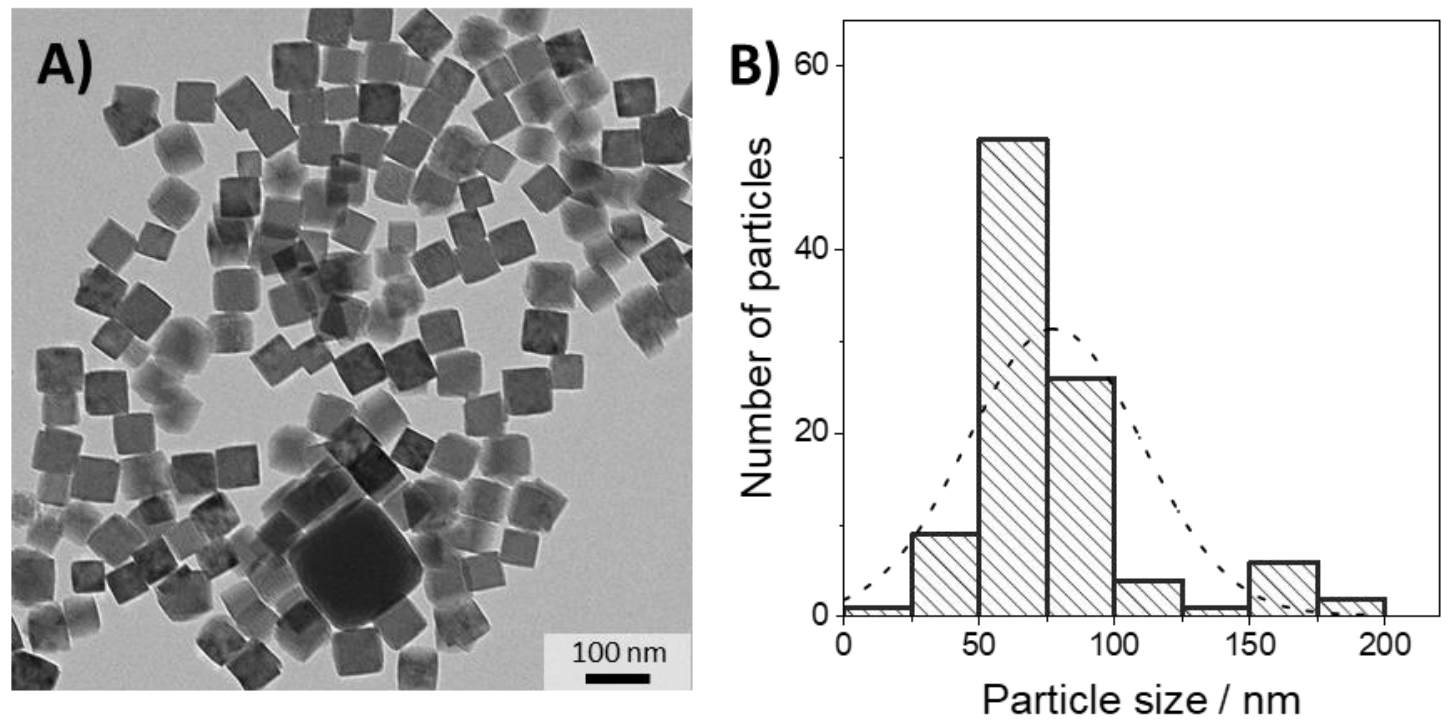

Figure S6. PBA-NiFe ${ }^{\mathrm{II}} \mathrm{NPs}$ and its corresponding histogram. 

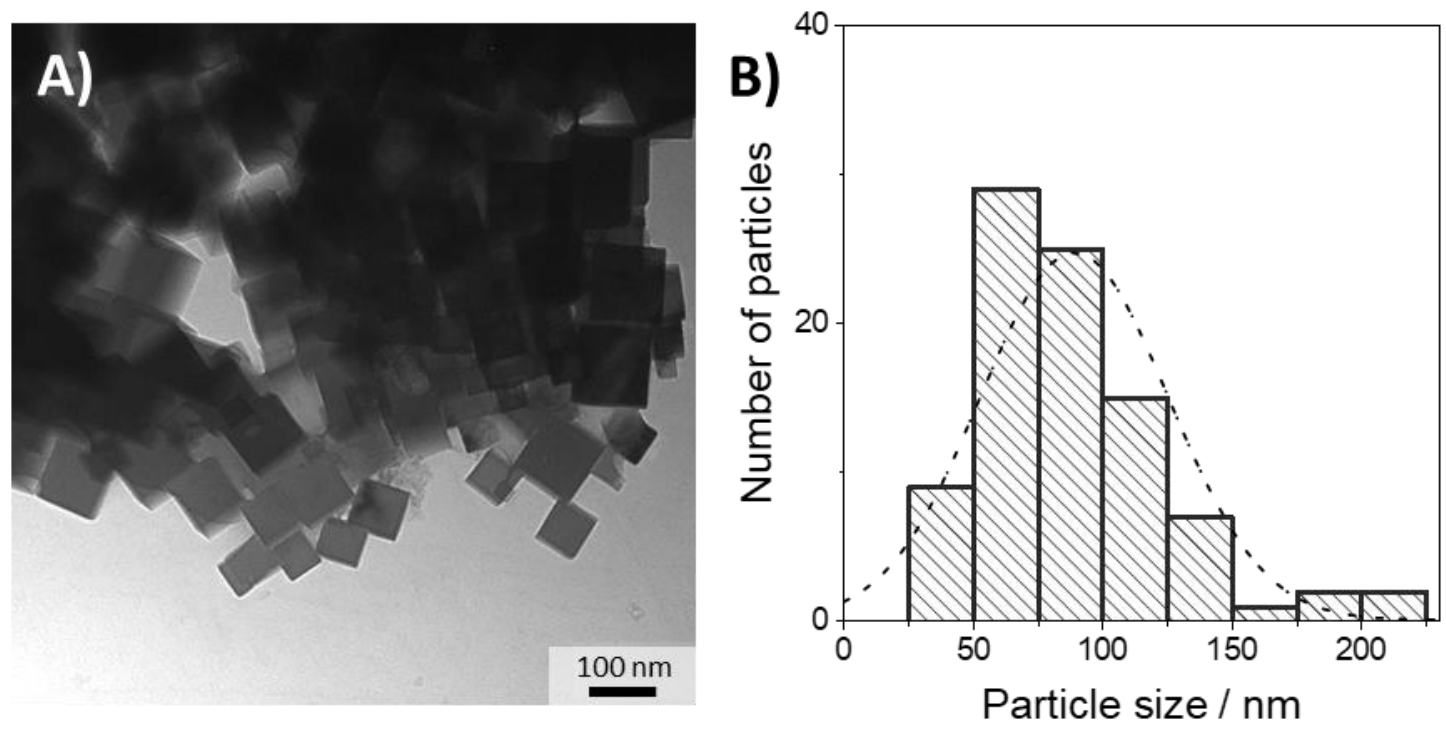

Figure S7. PBA-CoFe ${ }^{\mathrm{II}} \mathrm{NPs}$ and its corresponding histogram. 

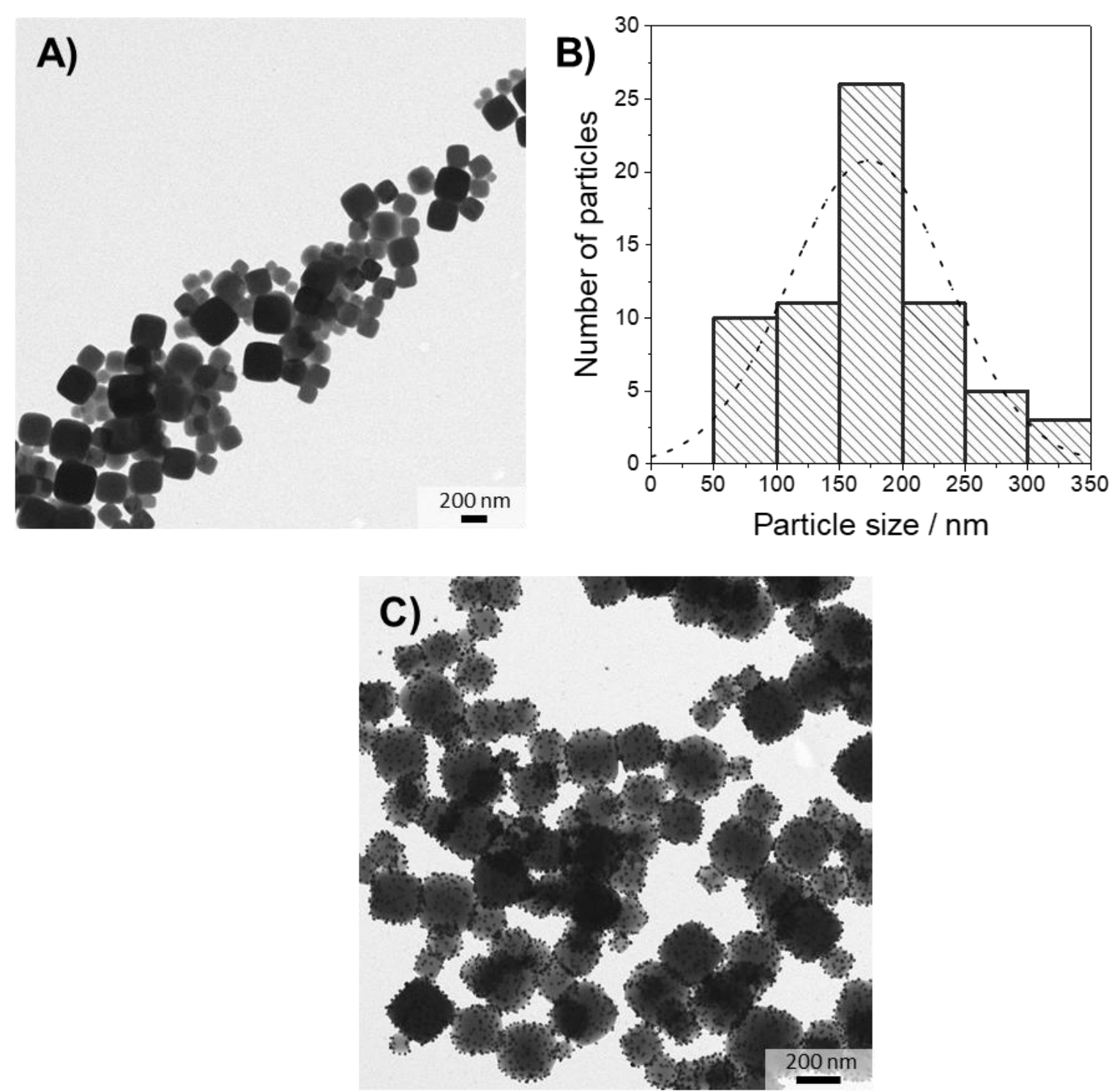

Figure S8. A) B) PBA-NiFe NPs and its corresponding histogram. C) Au-decorated PBA-NiFe NPs. 

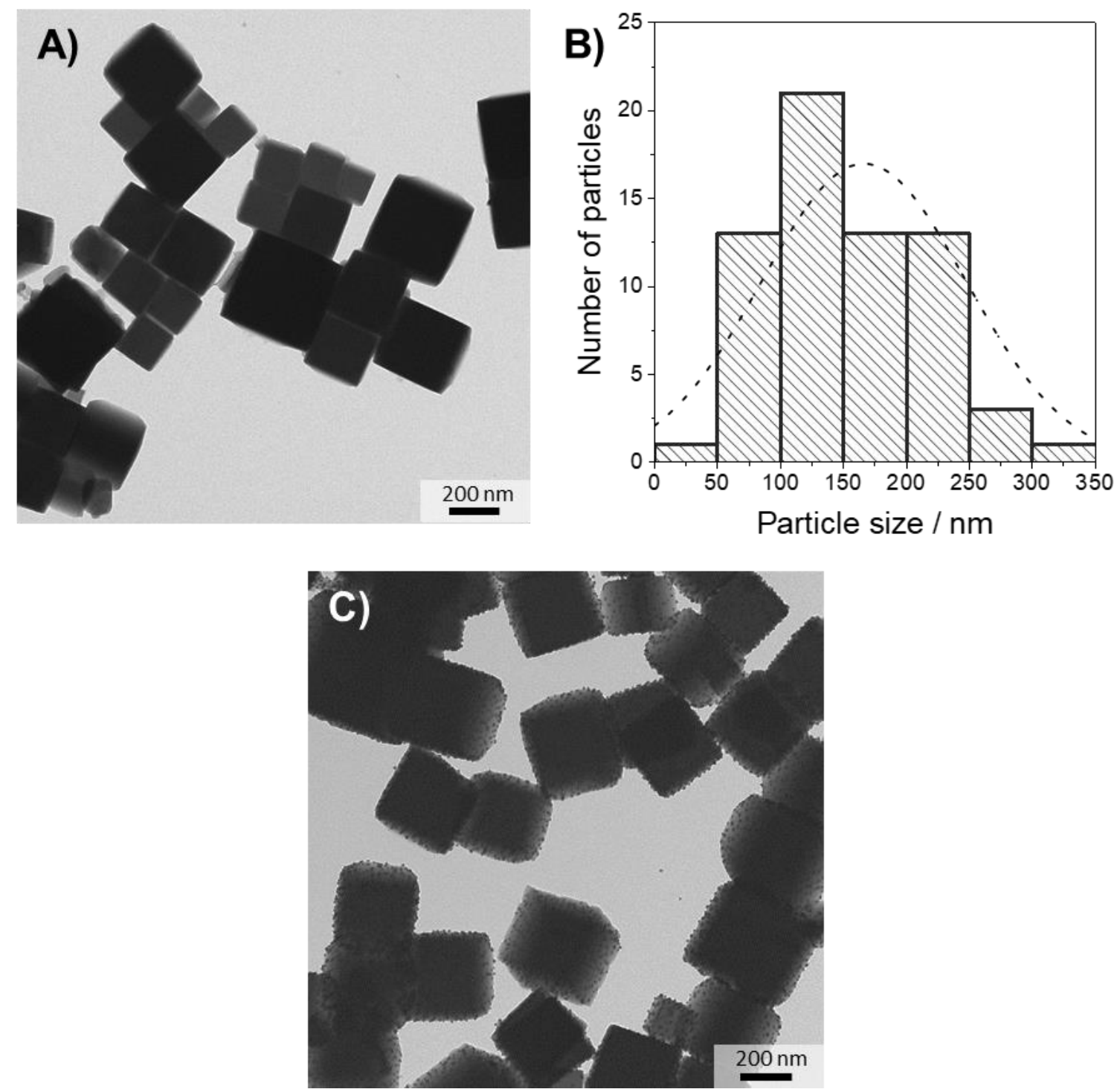

Figure S9. A) B) PBA-CoFe NPs and its corresponding histogram. C) Au-decorated PBACoFe NPs. 

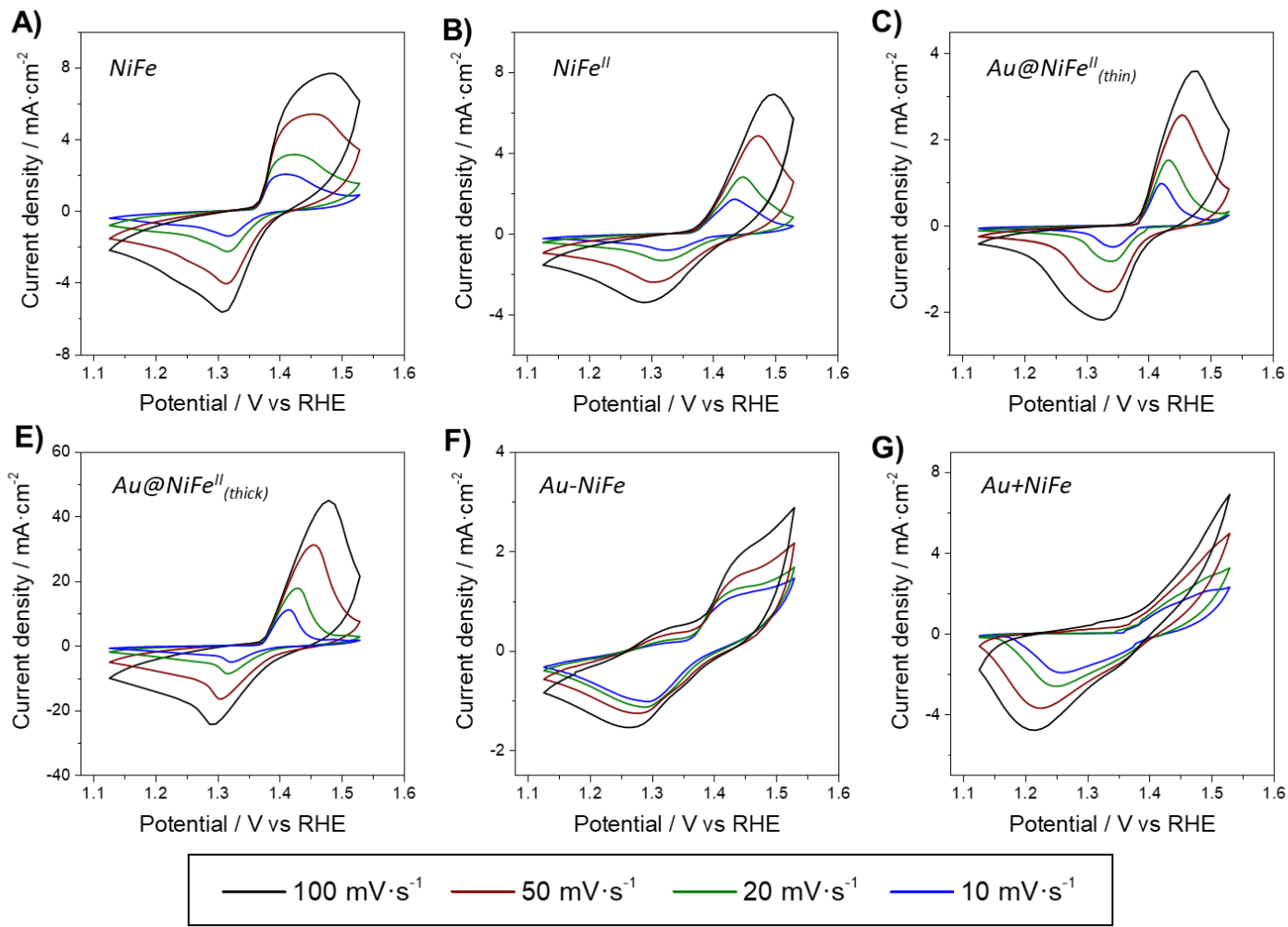

Figure S10. Voltammetric responses to potential cycles performed at different scan rates in 1 $\mathrm{M} \mathrm{KOH}$ aqueous solution for different NiFe nanoparticles: A) PBA, B) PBA with Fe ${ }^{\mathrm{II}}, \mathrm{C}$ ) Au@PBA (thin shell), D) Au@PBA (thick shell), E) Au decorated PBA NPs and F) physical mixture of Au and PBA NPs. 

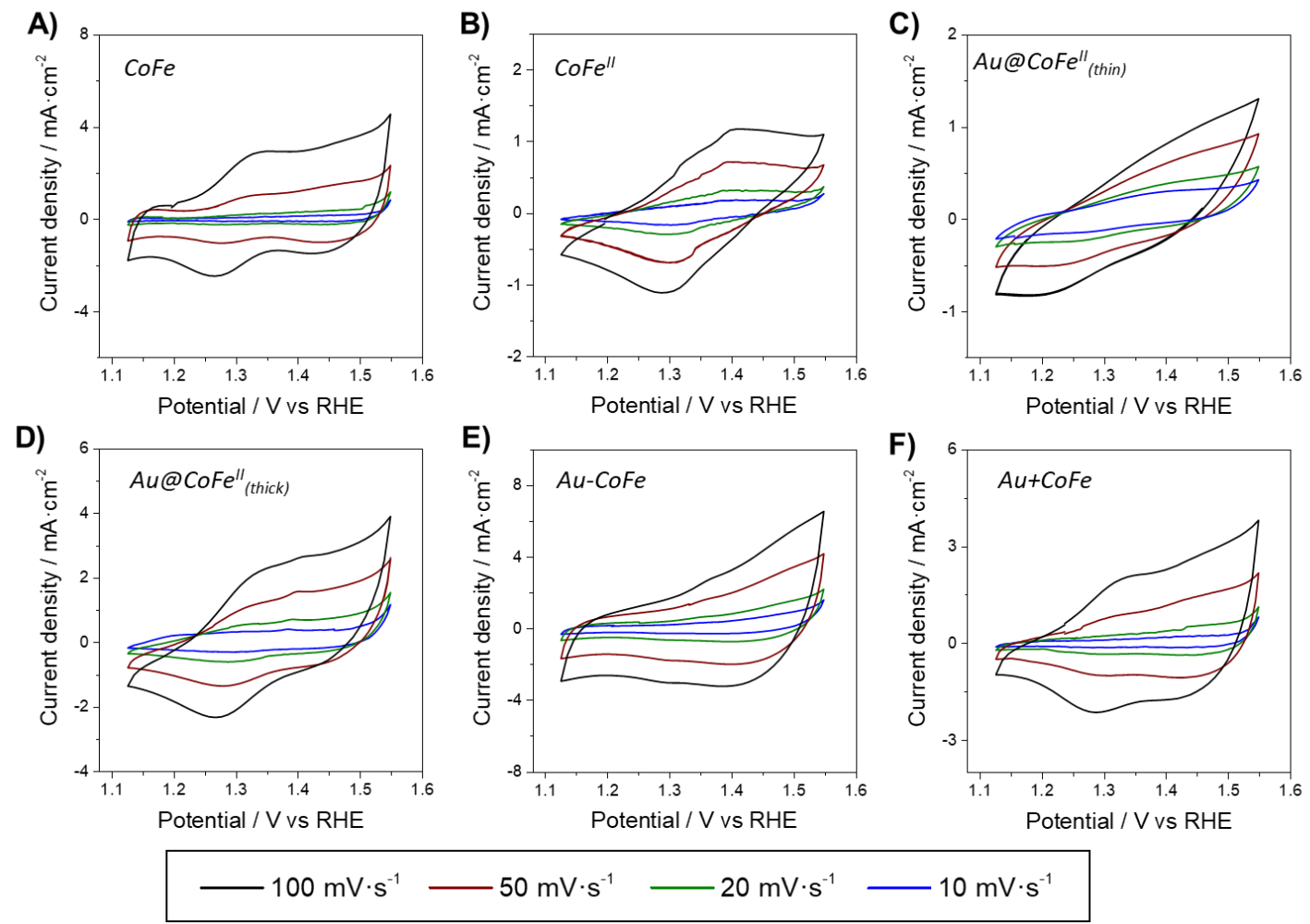

Figure S11. Voltammetric responses to potential cycles performed at different scan rates in 1 M KOH aqueous solution for different CoFe nanoparticles: A) PBA, B) PBA with Fe ${ }^{\text {II }}$ C) Au@PBA (thin shell), D) Au@PBA (thick shell), E) Au decorated PBA NPs and F) physical mixture of Au and PBA NPs. 

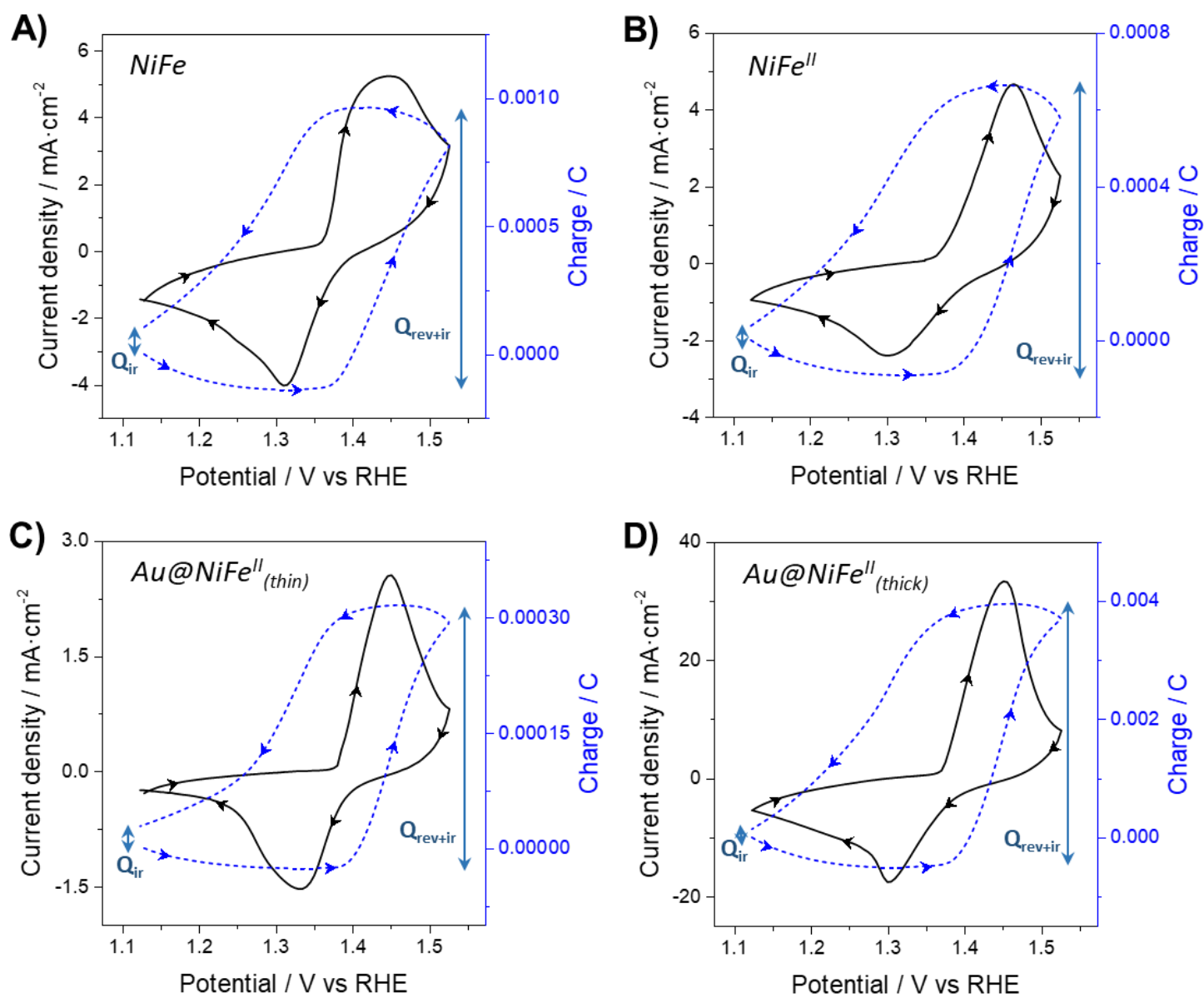

Figure S12. Evolution of the consumed charge (coulovoltammetric response) parallel to the voltammetric response to the cyclic voltammetry performed at $50 \mathrm{mV} \cdot \mathrm{s}^{-1}$ in $1 \mathrm{M} \mathrm{KOH}$ aqueous solution for $\mathrm{NiFe}$ compounds. 

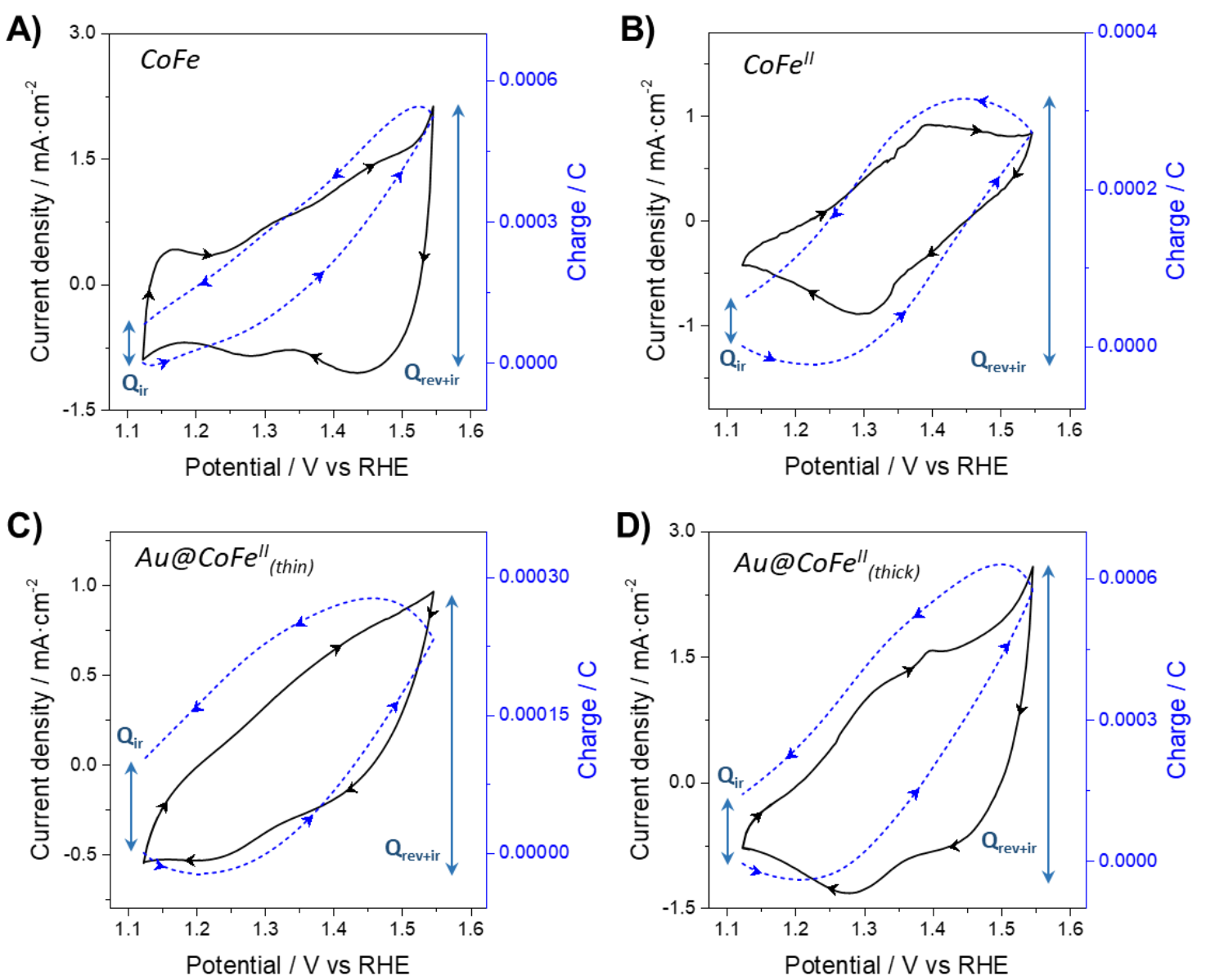

Figure S13. Evolution of the consumed charge during the voltammetric responses to potential cycles performed at $50 \mathrm{mV} \cdot \mathrm{s}^{-1}$ in $1 \mathrm{M} \mathrm{KOH}$ aqueous solution for CoFe compounds. 
Table S3. Amount of electroactive PBA mass being reversibly oxidized/reduced during cyclovoltamperometric experiments.

\begin{tabular}{|c|c|c|c|c|c|}
\hline \multirow[b]{2}{*}{ Samples } & \multirow{2}{*}{$\begin{array}{l}\text { PBA mass } \\
\left(\mathrm{mg} / \mathrm{cm}^{2}\right)\end{array}$} & \multicolumn{2}{|c|}{$z=1$} & \multicolumn{2}{|l|}{$z=2$} \\
\hline & & $\begin{array}{c}\text { PBA e.a. mass } \\
\left(\mathrm{mg} / \mathrm{cm}^{2}\right)\end{array}$ & $\begin{array}{c}\text { PBA e.a. } \\
\text { (\%) }\end{array}$ & $\begin{array}{c}\text { PBA e.a. mass } \\
\left(\mathrm{mg} / \mathrm{cm}^{2}\right)\end{array}$ & $\begin{array}{c}\text { PBA e.a. } \\
\text { (\%) }\end{array}$ \\
\hline NiFe & 0.25 & 0.026 & 10.2 & 0.013 & 5.1 \\
\hline $\mathrm{NiFe}^{\prime \prime}$ & 0.25 & 0.0178 & 6.4 & 0.009 & 3.2 \\
\hline$A u @ N i F e^{\prime \prime}{ }_{(\text {thin })}$ & 0.14 & 0.01 & 8 & 0.005 & 4 \\
\hline Au@NiFe & 0.23 & 0.102 & 42.4 & 0.051 & 21.2 \\
\hline CoFe & 0.25 & 0.0084 & 3.4 & & \\
\hline $\mathrm{CoFe}$ & 0.25 & 0.0071 & 2.6 & & \\
\hline$A u @ \mathrm{CoFe}_{(\text {thin) }}$ & 0.12 & 0.0044 & 3.2 & & \\
\hline$A u @ C o F e^{\prime \prime}{ }_{\text {(thick) }}$ & 0.24 & 0.0182 & 7.8 & & \\
\hline
\end{tabular}

The electroactive mass (e.a.) of PBA was estimated using Faraday's law of electrolysis:

$$
m=\frac{Q}{F} \cdot \frac{M}{Z}
$$

Being, $m$ the mass $(\mathrm{g})$, $\mathrm{Q}$ the charge corresponding to the reversible redox process, $\mathrm{M}$ the molecular weight of the sample and $\mathrm{z}$ the total number of electrons taking part in the redox process.

Q was calculated by subtracting the total charge involving reversible and irreversible reactions $\left(\mathrm{Q}_{\mathrm{rev}+\mathrm{ir}}\right)$ and the charge consumed by the irreversible oxygen evolution, $\left(\mathrm{Q}_{\mathrm{ir}}\right)$ calculated in Figures S12 and S13. The reversible charge allows the calculations of the number of electroactive metal atoms.

The redox processes of NiFe compounds are associated with the oxidation/reduction of Ni. Here, the initial $\mathrm{Ni}^{2+}$ is firstly oxidized to a mixed valance state of $\mathrm{Ni}^{3+} / \mathrm{Ni}^{4+}$. Therefore, calculations were carried out considering one $(\mathrm{z}=1)$ or two $(\mathrm{z}=2)$ electrons involved in the reaction.

For $\mathrm{CoFe}$ compounds, the redox processes associated with the reaction of $\mathrm{Co}^{2+}$ and $\mathrm{Co}^{3+}$ involves one electron $(\mathrm{z}=1)$. 

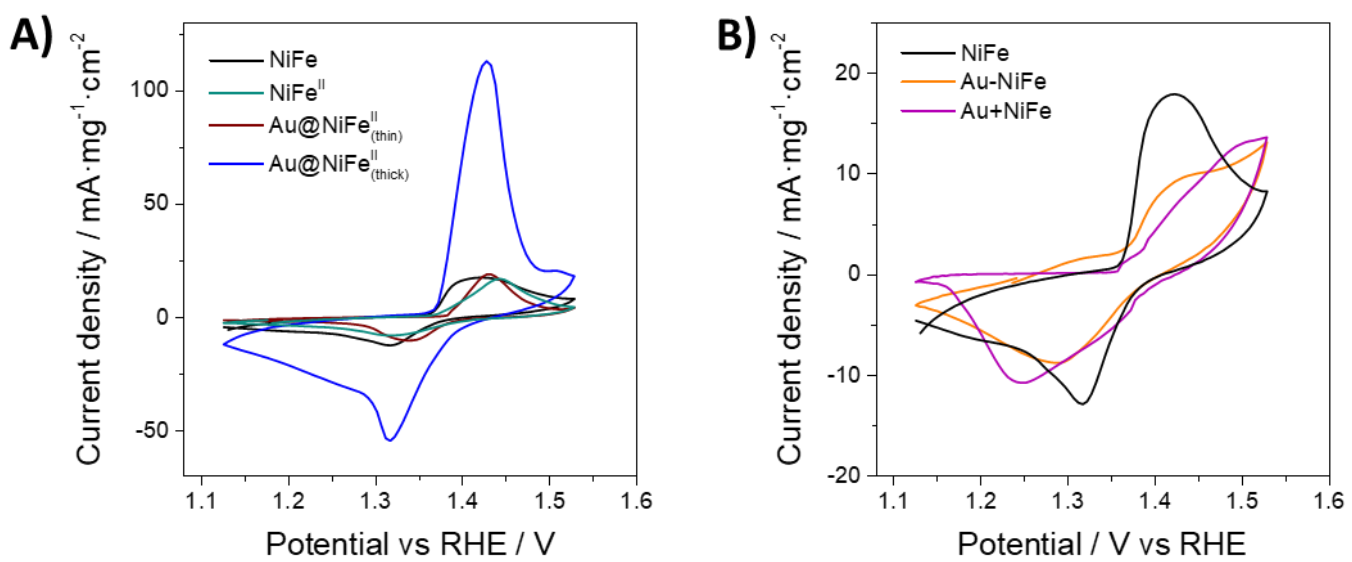

Figure S14. Voltammetric responses to potential cycles performed at $20 \mathrm{mV} \cdot \mathrm{s}^{-1}$ in $1 \mathrm{M} \mathrm{KOH}$ aqueous solution for $\mathrm{NiFe}$ compound compared to: $\mathrm{A}) \mathrm{NiFe}$ (II) and the electroactive species of $\mathrm{Au} @ \mathrm{NiFe}$ (thin) and $\mathrm{Au} @ \mathrm{NiFe}$ (thick); and $\mathrm{B}$ ) the electroactive species of $\mathrm{Au}-\mathrm{NiFe}$ and $\mathrm{Au}+\mathrm{NiFe}$. 

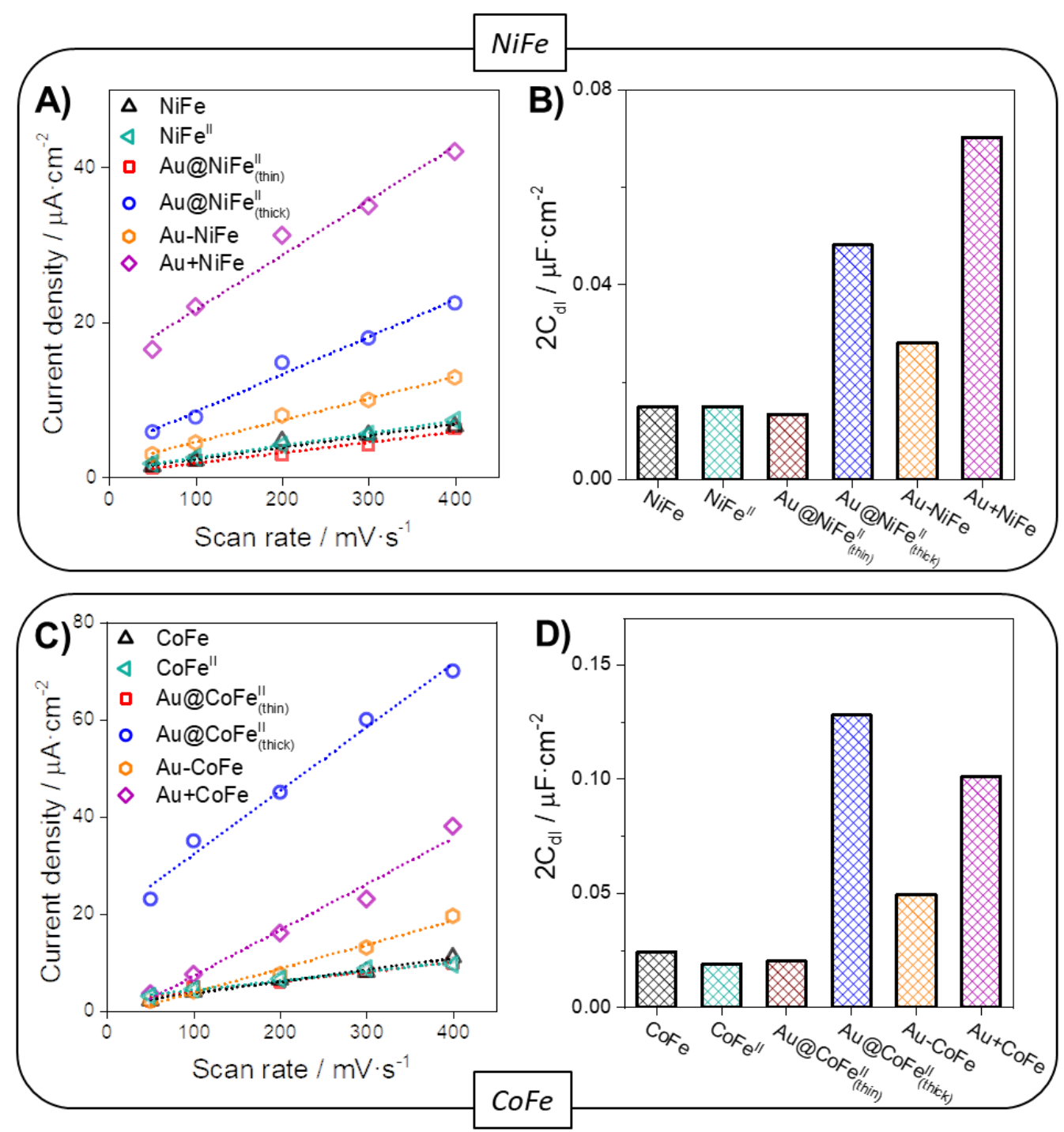

Figure S15. Linear slopes representing the ECSA calculated from CVs performed in a nonfaradaic region at different scan rates for $\mathrm{NiFe}(\mathrm{A})$ and $\mathrm{CoFe}(\mathrm{C}) \mathrm{PBA}$ compounds. ECSA values of the different NPs of $\mathrm{NiFe}(\mathrm{B})$ and $\mathrm{CoFe}(\mathrm{D}) \mathrm{PBA}$ compounds. 

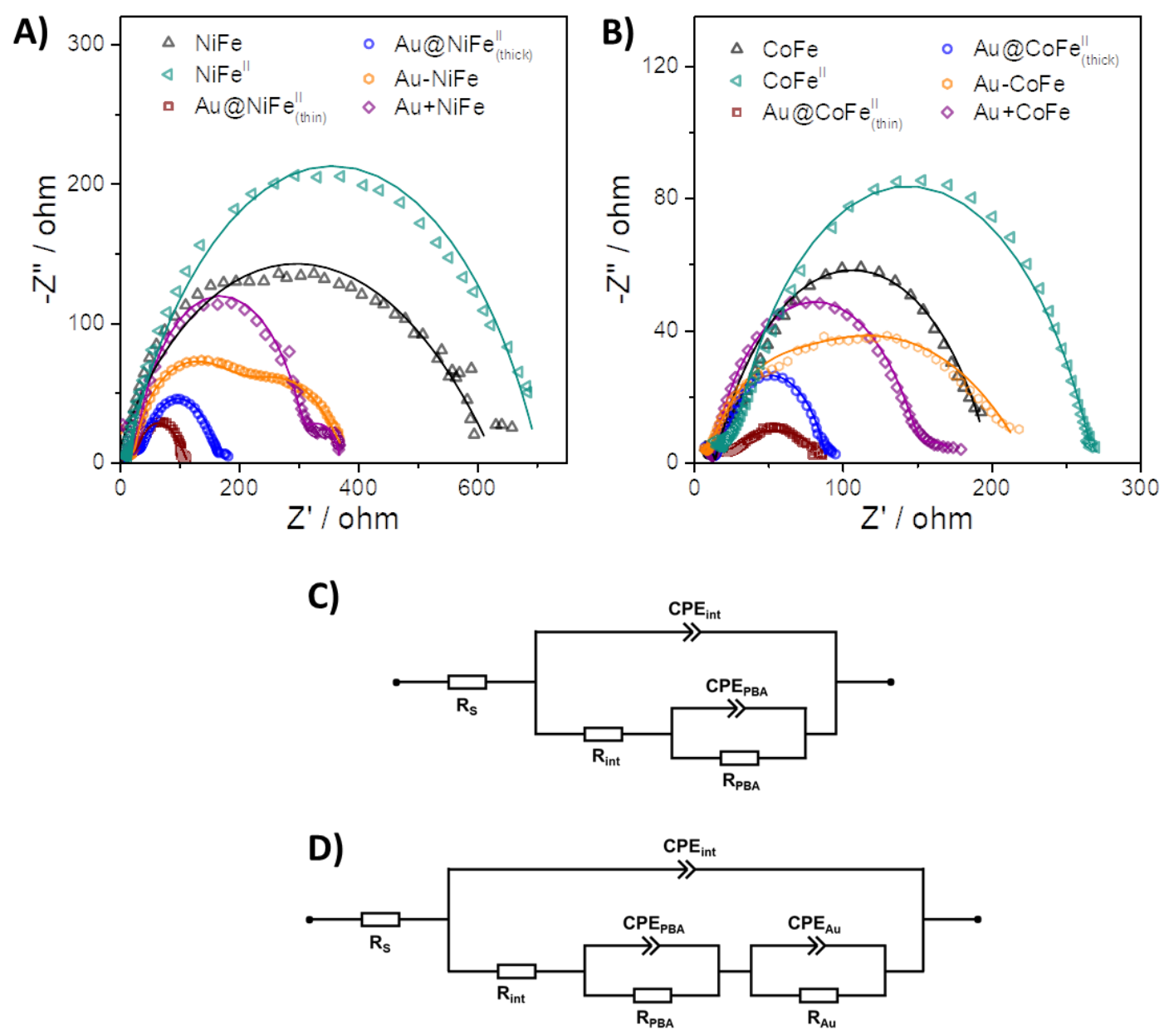

Figure S16. A) B) Nyquist plots of the different samples recorded at an overpotential of $0.4 \mathrm{~V}$. Points correspond to experimental data, and lines are curves fitted with the equivalent circuit. C) Equivalent circuit used for the two PBA NPs, Au@PBA(thin) and Au@PBA(thick). D) Equivalent circuit used for Au-PBA and Au+PBA. 
Table S4. Comparison of the electrocatalytic activity with previous reported OER electrocatalysts.

\begin{tabular}{|c|c|c|c|c|c|}
\hline Sample & $\begin{array}{c}\text { Overpotential at } 10 \\
\mathbf{m A} \cdot \mathbf{c m}^{-2}(\mathrm{~V} \text { vs RHE})\end{array}$ & Solution & iR correction & Electrode & Reference \\
\hline$A u @ N i F e$ & 1.59 & $1 \mathrm{M} \mathrm{KOH}$ & no & $\mathrm{GC}$ & This work \\
\hline$A u @ C o F e$ & 1.57 & $1 \mathrm{M} \mathrm{KOH}$ & no & $\mathrm{GC}$ & This work \\
\hline $\mathrm{Au} @ \mathrm{CoO}_{4}$ & 1.61 & $0.1 \mathrm{M} \mathrm{KOH}$ & yes & GC & {$[16]$} \\
\hline$A u @ \mathrm{CoFeO} \mathrm{O}_{x}$ & 1.55 & $1 \mathrm{M} \mathrm{KOH}$ & yes & $\mathrm{GC}$ & {$[48]$} \\
\hline$A u @ N i O$ & 1.63 & $1 \mathrm{M} \mathrm{KOH}$ & yes & GC & {$[48]$} \\
\hline CoFe-LDH & 1.65 & $1 \mathrm{M} \mathrm{KOH}$ & no & $\mathrm{GC}$ & {$[45]$} \\
\hline $\mathrm{NiFe}-\mathrm{LDH}$ & 1.58 & $1 \mathrm{M} \mathrm{KOH}$ & yes & $\mathrm{GC}$ & {$[58]$} \\
\hline $\mathrm{IrO}_{2}$ & 1.57 & $1 \mathrm{M} \mathrm{KOH}$ & yes & $\mathrm{GC}$ & {$[58]$} \\
\hline 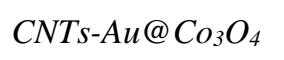 & 1.58 & $1 \mathrm{M} \mathrm{KOH}$ & no & GC & [49] \\
\hline $\mathrm{NiFe}-V_{C N-}$ & 1.513 & $1 \mathrm{M} \mathrm{KOH}$ & yes & RDE GC & {$[38]$} \\
\hline $\mathrm{CoFe}-V_{C N-}$ & 1.599 & $1 \mathrm{M} \mathrm{KOH}$ & yes & RDE GC & {$[38]$} \\
\hline $\mathrm{ZnCo}_{2} \mathrm{O}_{4} / \mathrm{Au} / \mathrm{CNTs}$ & 1.67 & $1 \mathrm{M} \mathrm{KOH}$ & no & RDE GC & {$[46]$} \\
\hline $\mathrm{NiO}-\mathrm{NiFe}_{2} \mathrm{O}_{4} / r G O$ & 1.53 & $1 \mathrm{M} \mathrm{KOH}$ & no & RDE GC & {$[53]$} \\
\hline CoFe oxide & 1.54 & $1 \mathrm{M} \mathrm{KOH}$ & yes & $\mathrm{Ni}$ foam & {$[59]$} \\
\hline CoFe film & 1.66 & $0.1 \mathrm{M} \mathrm{KOH}$ & no & FTO glass & [9] \\
\hline$A u / N i F e-L D H$ & 1.467 & $1 \mathrm{M} \mathrm{KOH}$ & yes & Ti mesh & {$[50]$} \\
\hline$A g @ \mathrm{Co}(\mathrm{OH})_{2}$ & 1.48 & $1 \mathrm{M} \mathrm{KOH}$ & no & Carbon cloth & {$[51]$} \\
\hline $\mathrm{Ag}+\mathrm{Co}(\mathrm{OH})_{2}$ & 1.55 & $1 \mathrm{M} \mathrm{KOH}$ & no & Carbon cloth & {$[51]$} \\
\hline
\end{tabular}



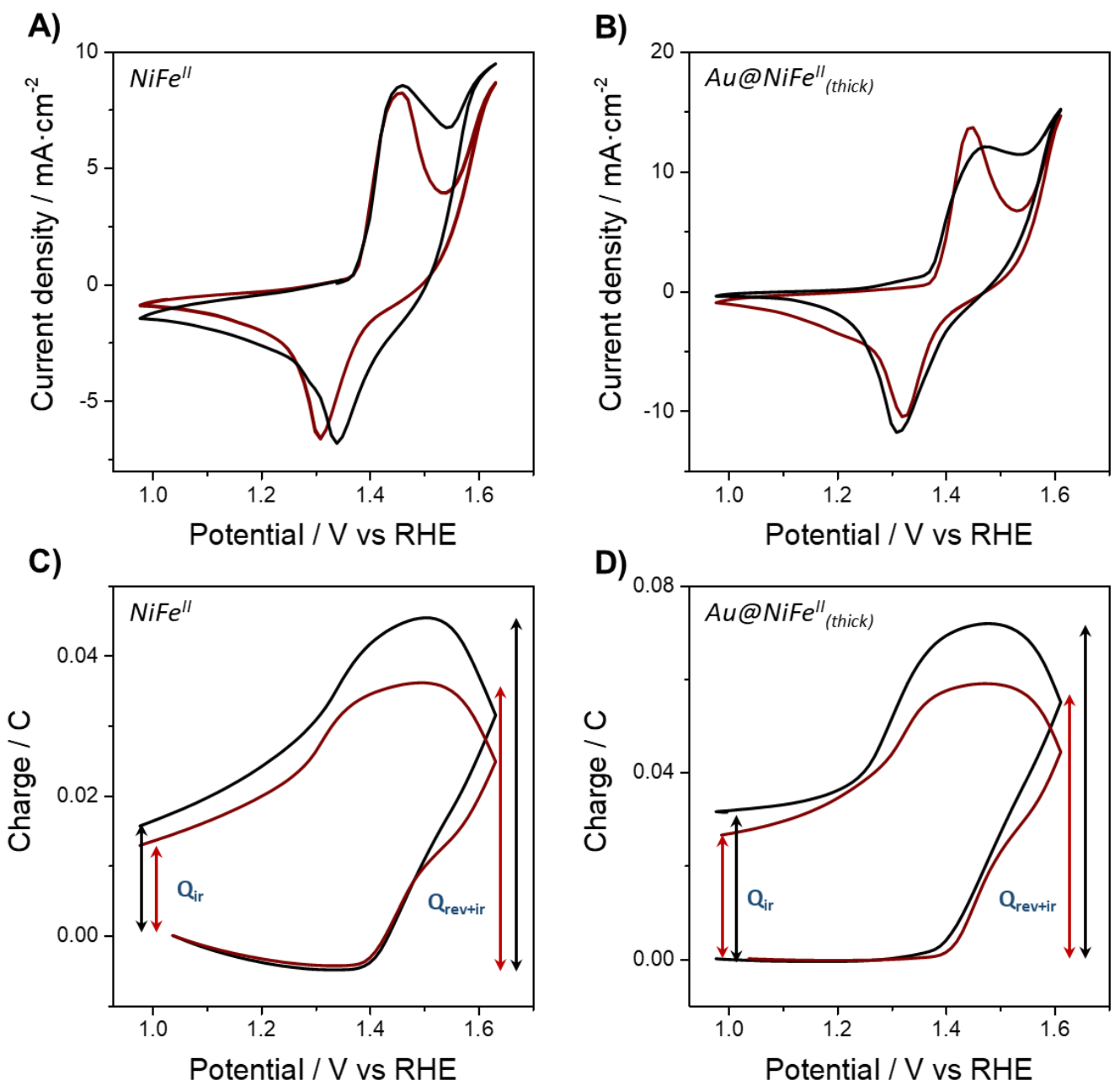

Figure S17. A) B) Voltammetric responses to potential cycles performed at $50 \mathrm{mV} \cdot \mathrm{s}^{-1}$ in $1 \mathrm{M}$ $\mathrm{KOH}$ aqueous solution and $\mathrm{C}$ ) $\mathrm{D}$ ) evolution of the consumed charge (coulovoltammetric response) parallel to the voltammetric response to the cyclic voltammetry performed at 50 $\mathrm{mV} \cdot \mathrm{s}^{-1}$ for $\mathrm{NiFe}^{\mathrm{II}}$ and $\mathrm{Au} @ \mathrm{NiFe}($ thick) compounds before and after stability test. Black colors are referred to measurements before stability test and red colors are referred to measurements after stability test. 

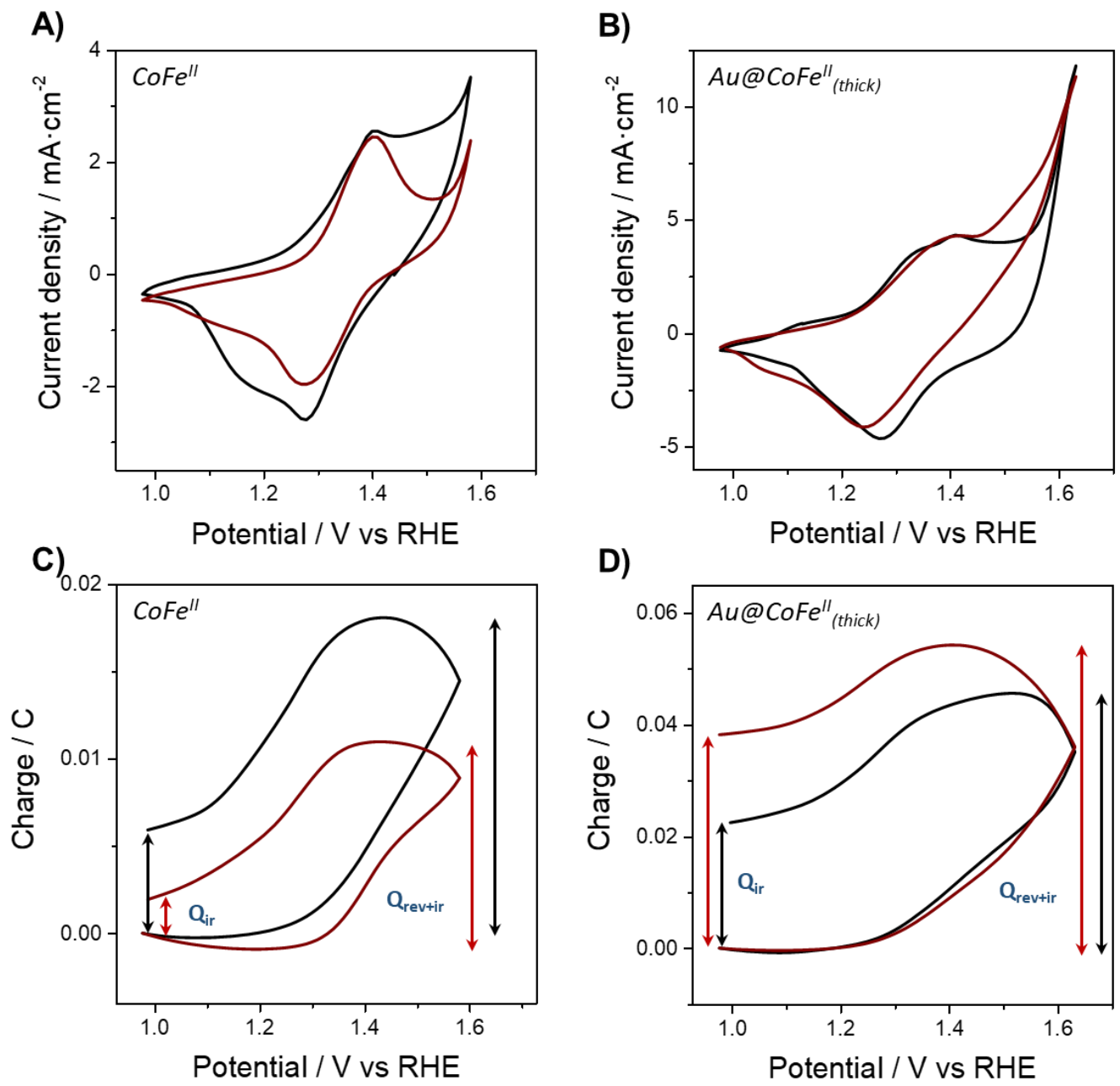

Figure S18. A) B) Voltammetric responses to potential cycles performed at $50 \mathrm{mV} \cdot \mathrm{s}^{-1}$ in $1 \mathrm{M}$ $\mathrm{KOH}$ aqueous solution and $\mathrm{C}$ ) $\mathrm{D}$ ) evolution of the consumed charge (coulovoltammetric response) parallel to the voltammetric response to the cyclic voltammetry performed at 50 $\mathrm{mV} \cdot \mathrm{s}^{-1}$ for $\mathrm{CoFe} \mathrm{II}^{\mathrm{II}}$ and $\mathrm{Au} @ \mathrm{CoFe}$ (thick) compounds before and after stability test. Black colors are referred to measurements before stability test and red colors are referred to measurements after stability test. 